\title{
A Comparative Evaluation of Indirect Sequence-Specific Magneto- extraction-aided Fluorescence and Electrochemical LAMP with SARS-CoV-2 Nucleic Acid as the Analyte
}

Sayantan Tripathy ${ }^{\text {abd\# }}$, Tanvi Agarkar ${ }^{\mathrm{c} \#}$, Arunansu Talukdar ${ }^{\mathrm{e}}$, Mrittika Sengupta ${ }^{\text {bd }}$, Ashvani Kumar $^{\text {cf* }}$, Souradyuti Ghosh ${ }^{\text {abd** }}$

a, Department of Chemistry, Bennett University, India

b, Department of Biotechnology, Bennett University, India

c, Department of Physics, Bennett University, India

d, Center of Excellence for Nanosensors and Nanomedicine, Bennett University, India

e, Geriatric Medicine Department, Medical College and Hospital, Kolkata, India

f, Pristine Diamonds Pvt. Ltd, Vatva, India

\#, joint first author.

*, Corresponding author.

**, Corresponding author, will handle communications (souradyuti.ghosh@bennett.edu.in) 
Abstract. Since the beginning of the SARS-CoV-2 pandemic, nucleic acid amplification test (NAAT) such as quantitative real-time reverse transcriptase PCR (qRT-PCR) has remained the primary intervention for diagnostics and containment of SARS-CoV-2. Despite its remarkable clinical as well as analytical specificity and sensitivity, qRT-PCR necessitates pure nucleic acid free of any polymerase inhibitors (from complex biological matrices) as its substrate. Similarly, isothermal NAATs (iNAATs), despite their advantage over qRT-PCR in terms of thermal cycler independence, still require pure nucleic acid as a template. The requirement of pure nucleic acid in turn warrants the use of spin-column mediated extraction with centralized high-speed centrifuges. Additionally, utilization of centralized real-time fluorescence readout and use of sequence-specific molecular probes like TaqMan further prevent their deployment in decentralized locations. To circumvent these disadvantages, we have envisioned a sample-to-answer workflow comprising of indirect sequence-specific magneto-extraction (also referred to as magnetocapture, magneto-preconcentration, or magneto-enrichment in this manuscript) followed by in situ fluorescence or electrochemical LAMP. This study, using SARS-CoV-2 nucleic acid as the analyte, compared the analytical effectiveness of indirect and direct sequence-specific magnetoextraction followed by LAMP. Since contamination carryover may affect the efficacy of sequencespecific indirect magnetocapture, its performance in presence of excess host nucleic acid or serum was probed. Through these experiments, we have established a comprehensive limited resourceadoptable and highly specific nucleic acid detection method with the limit of detection of 2.5 copies/ $\mu \mathrm{L}$. Its advantage lies in the flexibility of using either centralized real-time SYBR-based fluorescence LAMP or portable electrochemical LAMP as the readout. Simultaneously, the performance with magneto-capture aided fluorescence and electrochemical LAMP readouts were weighed against each other in terms of analytical sensitivity, specificity, and turnaround time. 
Additionally, the analytical efficacy of the magnetocapture-LAMP workflow was also checked against that of LAMP using pure nucleic acid as a template. Besides being the first report utilizing electrochemical LAMP to detect SARS-CoV-2 nucleic acid, this would probably be the first study to make the analytical comparative assessment of magnetic preconcentration combined with in situ fluorescence and electrochemical LAMP. It is probably also the first study (to the best of our knowledge) to compare the analytical efficacy of a sequence-specific magnetic target enrichmentLAMP (fluorescence and electrochemical) to that of a LAMP assay using pure nucleic acid as a template.

KEYWORDS: Magnetic sequence-specific target enrichment; Quantitative real-time LAMP; Electrochemical LAMP; SARS-CoV-2.

Abbreviations: human genomic DNA, hgDNA; quantitative real time reverse transcriptase PCR, qRT-PCR; quantitative real time LAMP, qLAMP; quantitative real time reverse transcriptase LAMP, qRT-LAMP; electrochemical end-point LAMP (eLAMP); electrochemical reverse transcription end-point LAMP, eRT-LAMP; nucleic acid amplification technique, NAAT; viral transmission media, VTM. 


\section{Introduction:}

The upsurge of novel corona virus disease (COVID-19) is caused by the emergence of the severe acute respiratory syndrome-2 virus, commonly known as SARS-CoV-2. Starting in December 2019 in Wuhan city, China ${ }^{1}$, the outbreak then quickly spread to the rest of the world. Till date (January 2022) more than 351 million people have been infected with this virus with 5.6 million deaths ${ }^{6}$. Transmission of SARS-CoV-2 is known to spread via close contact with another infected person ${ }^{7}$ or through saliva or respiratory secretions expelled from the former during coughs, sneezes, talks ${ }^{7}$. While an infected person showing typical symptoms (fever, cough, or breath shortness) could be rapidly quarantined or admitted for medical care, an asymptomatic patient, if undiagnosed, can also spread the infection to healthy individuals. Due to severe limitations of proper diagnostics infrastructure, the population at the limited resource settings are more vulnerable to the onslaught of the disease ${ }^{8}$. Furthermore, the requirement of sample transport from limited-resource settings to centralized diagnostic labs increases both the cost and turnaround time, amplifying the disease burden. Therefore, fast, felicitous, and near-point-of-care (near-POC) testing for SARS-CoV-2 is the foremost demand for virus detection and prompt management of this pandemic.

The SARS-CoV-2, like other coronaviruses, could be detected via the quantitative reverse transcription-polymerase chain reaction (qRT-PCR) using molecular targets present in their genome $^{9}$. Despite the widespread adoption of real-time PCR instruments, the upfront cost of instrument procurement and the necessity of highly trained personnel hinder its deployment to resource-constrained areas. On the other hand, serological tests involving $\mathrm{IgG} / \mathrm{IgM}$ detection suffer from low analytical sensitivity early in the infection ${ }^{10}$. Similarly, antigen-targeting lateral flow assays also have inadequate analytical sensitivity $\left(10^{6}\right.$ copies $/ \mathrm{mL}$ in the real-life scenario), leading 
to low sensitivity $(65-78 \%)$ and false-negative detection ${ }^{11,12}$. Therefore, most of the national medical bodies, including the Center for Disease Control and Prevention (CDC $)^{13}$, recommend qRT-PCR as the gold-standard method of detecting SARS-CoV-2 due to its high specificity and sensitivity. Despite the availability of several commercial qRT-PCR kits, however, there are several general disadvantages of it. The qRT-PCR requires pure nucleic acid (and therefore prior nucleic acid extraction from the biological samples), which increases the overall turnaround time, cost, and need for a high-speed centrifuge usually present in a centralized lab. In addition, a separate reverse transcription step is often required as very few existing kits integrate the reverse transcription with the real time PCR step. In fact, single-step qRT-PCR has lower sensitivity than that of two-step assays ${ }^{14,15}$. The multi-step assays also necessitate the recruitment of highly trained manpower for conducting them. In addition, the possible presence of host nucleic acid requires a sequence-specific TaqMan or molecular beacon probe in the assay to enhance specificity, once again adding to the cost.

To circumvent some of these drawbacks of real-time PCR, isothermal nucleic acid amplification techniques (iNAATs) such as loop-mediated isothermal amplification (LAMP) have been employed to detect the viral nucleic acid ${ }^{16}$. Without needing a thermal cycler, LAMP provides nucleic acid amplification with a long DNA concatemer as an amplicon in less than 30 minutes using $4-6$ primers and a thermophilic strand displacement DNA polymerase (Bacillus stearothermophilus or Bst polymerase and its variants $)^{17}$. Unlike PCR for RNA targets, reverse transcription LAMP (RT-LAMP) amplification can be carried out in a single step through the simultaneous addition of a reverse transcriptase enzyme. In SARS-CoV-2 nucleic acid detection using LAMP, strategies such as phenol-red based colorimetric RT-LAMP ${ }^{18}$, a two-step closed tube LAMP strategy using fluorometric readout ${ }^{19}$, SARS-CoV-2 DNA Endonuclease-Targeted 
CRISPR Trans Reporter (DETECTR) coupled with LAMP ${ }^{20}$, and a LAMP strategy combined with a gene finder dye integrated fluorescence-based tool has ben utilized ${ }^{21}$. Besides such real-time fluorescence or colorimetric readout, a LAMP reaction could in principle be detected by end-point detection of the amplicons using a portable electrochemical biosensor ${ }^{22}$. While providing quantitative readouts in laboratory settings, this feature could be utilized in NAAT diagnostics in a resource-limited area as well ${ }^{22}$. Despite such advantages and sensitivity of LAMP, most of the aforementioned assays require prior RNA extraction from viral transmission media (VTM). In fact, very few studies (with or without concerning SARS-CoV-2 detection) have been reported where separate nucleic acid extraction process has been bypassed or integrated with downstream in situ LAMP or other NAATs ${ }^{16}$. On the contrary, it has been demonstrated that NAAT using nonextracted nucleic acid as a template significantly reduced the specificity and sensitivity for SARSCoV-2 detection and may not work at all with SYBR-based qRT-PCR ${ }^{23}$. Apart from this, the existing studies have not demonstrated whether or how efficiently LAMP would detect target nucleic acid in presence of excess host nucleic acid. Uses of molecular beacon probe to improve LAMP specificity in presence of host nucleic acid would limit the cost-efficacy for the assay. Besides, no studies have surprisingly been done yet on SARS-CoV-2 LAMP amplification with electrochemical readouts, despite their advantageous utility in the resource-constrained area through portability, easy operation, and low cost.

A magnetic pre-concentration to purify target nucleic acid is advantageous compared to spin-column-based extraction for NAAT diagnostics' sample-to-answer workflow. This is due to the former's minimal equipment necessity, the scope of in situ amplification, lesser turn-around time, and reduced $\operatorname{cost}^{24}$. Such assays, in combination with electrochemistry-mediated readout, would be of high utility in diagnostics in limited-resource settings. The magnetic preconcentration 
can be broadly categorized into three types; whole nucleic acid extraction ${ }^{25}$, sequence-specific capture of target nucleic acid ${ }^{26}$, and immunomagnetic capture coupled with cell lysis ${ }^{27}$. The sequence-specific capture could be direct and indirect in nature and would help concentrate only target nucleic acid as opposed to whole nucleic acid extraction. Therefore, it would (in principle) eradicate the need for TaqMan or other sequence-specific reporter probes in the downstream NAAT assay. In the direct capture, a magnetic bead-immobilized sequence-specific probe exploits complementarity to extract target nucleic $\operatorname{acid}^{26}$. The indirect magneto-enrichment utilizes a sequence-specific probe to first bind to the target of interest prior to magnetic bead (alternatively, to a solid phase) immobilization ${ }^{28}$. Despite having superior analytical sensitivity, indirect magnetopreconcentration is also considered more vulnerable (compared to direct magnetopreconcentration assays) to the presence of host nucleic acid (from non-specific annealing) and polymerase inhibitors (due to absence of blocking) $)^{28,29}$.

Despite the potential advantage and utility of sequence-specific magneto-enrichment, a comprehensive literature search of magnetic extraction methods combined with LAMP tabulated in Table S1 yielded only four existing studies involving direct sequence-specific magnetoenrichment (entries 20, 23, 43, 46) ${ }^{26,30-32}$. All four methods utilized microfluidic setup, requiring additional flow-controller equipments (thereby adding to the cost). The rest of the methods predominantly are either whole nucleic acid extraction or immunomagnetic capture followed by lysis. The former also included all magneto-extraction associated SARS-CoV-2 detection techniques using LAMP (entries 7, 10, 14, 24, 35, 48, Table $S 1^{25,33-37}$ ). Surprisingly, none of these studies listed in Table S1 utilized electrochemical readout despite its promising potential in limited resource detection. Furthermore, the studies also did not employ indirect sequence-specific magneto-enrichment despite its superior analytical sensitivity (Table S1). In fact, none of the 
published magneto-extraction assisted NAAT methods for detecting SARS-CoV-2 has recruited either a sequence-specific capture (direct or indirect) in combination with or without an electrochemical readout ${ }^{34,38-43}$. Therefore, the modality of a sequence-specific indirect magnetoenrichment of pathogen nucleic acid (SARS-CoV-2 or otherwise) from a complex biofluid or host nucleic acid-containing sample with downstream LAMP, either with fluorescence or electrochemical readout, remains unexplored.

This work aims to establish a comprehensive sample-to-answer workflow that would be selectively able to extract target nucleic acid from samples potentially containing complex biofluid and host nucleic acid in a minimally instrument-intensive manner. It would then detect and quantify the same using an iNAAT with the possibility for low-cost, limited-resource-friendly deployment. With this goal, we have here probed the analytical performance of an indirect sequence-specific magneto-extraction followed by fluorescence (SYBR-based) and electrochemical LAMP detections using SARS-CoV-2 RdRp plasmid DNA and RNA as analytes. In doing so, we first compared the effectiveness of direct and indirect sequence-specific magnetoextraction using quantitative real-time LAMP (qLAMP). Next, the indirect magneto-extractionqLAMP assay was employed to detect SARS-CoV-2 nucleic acid from samples containing excess human genomic DNA (hgDNA) and serum to determine its analytical sensitivity and specificity in a real life-mimicking scenario. After optimizing electrochemical end-point LAMP (eLAMP) with pure nucleic acid, its compatibility with sequence-specific indirect magneto-extraction for detecting SARS-CoV-2 nucleic acid from real life-simulating samples was then ascertained. In conducting these experiments, we have qualitatively and quantitatively addressed three critical questions highly relevant for limited resource diagnostics. First, we evaluated how the presence of potential contaminants from serum and hgDNA would affect the analytical performance of a 
sequence-specific magnetocapture combined with downstream LAMP. Next, we investigated whether a sequence-specific magneto-extraction integrated with an eLAMP would have equivalent analytical sensitivity with magneto-extraction having a qLAMP readout. Finally, our experiments explored how both these assays would compare with qLAMP, eLAMP, or qRT-PCR assays using the pure nucleic acid template in terms of cost, turnaround time, analytical sensitivity, and specificity.

\section{Results and Discussion}

Use of plasmid construct containing SARS-CoV-2 RdRp gene and selection of LAMP primers

As per NIH Guideline for SARS-CoV-2 research, in vitro expression of partial viral RNA or protein from plasmid construct results in non-infectious products, permitting the experiments to be conducted under BSL-2 containment ${ }^{44,45}$. Similarly, bacteria carrying such plasmids in frozen glycerol stocks or culture could also be handled under BSL-1 as such bacteria or plasmids themselves are non-infectious. Accordingly, we have utilized plasmid expressing SARS-CoV-2 RNA-dependent RNA polymerase $(R d R p)$ gene and it's in vitro transcribed RNA in this study (plasmid \#14567, https://www.addgene.org/145671/). Three sets of published LAMP primers (described in Supporting Information Section 2) against the $R d R p$ gene were investigated for their ability to differentiate $10^{3}$ copies of the plasmid from no template control (NTC) in a quantitative real time LAMP (qLAMP) assay ${ }^{46}$. While all three successfully differentiated the template amplification from NTC in our hand, primer set 2 displayed the highest $\Delta \mathrm{C}_{t}\left(\mathrm{C}_{\mathrm{t}}\right.$ refers to cycle threshold) and therefore were utilized for the succeeding experiments ${ }^{46}$ (Figure S1A and B). Additionally, the limit of detection (LoD) study performed on this plasmid using quantitative realtime LAMP (qLAMP) with primer set 2 detected the analytical sensitivity at 10 copies/reaction 
(Figure S2). Using plasmid DNA as the target, the $\mathrm{C}_{\mathrm{t}}$ values in qLAMP reaction matched that of the original paper ${ }^{46}$.

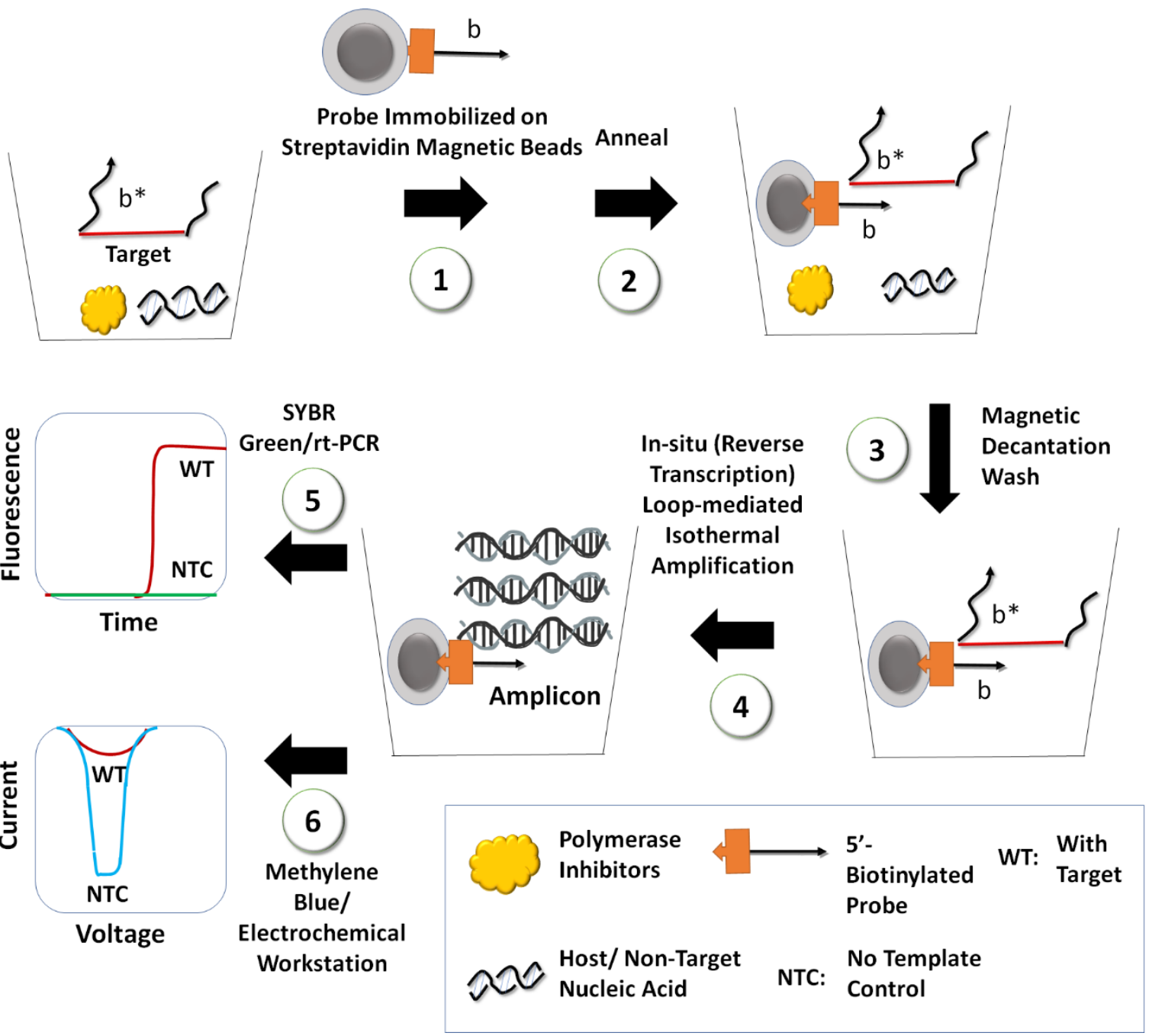

Scheme 1. Direct sequence-specific magnetocapture of nucleic acid by immobilizing the biotinylated probe on the magnetic beads followed by incubation with target (complementary to the biotinylated probe) (steps 1 and 2). The target nucleic acid could be present with host nucleic acid and polymerase inhibitors from complex biofluid. After magnetic decantation wash (step 3), the target bound magnetic beads was used for in situ LAMP (or reverse transcription LAMP, step 4) amplification detected by quantitative real-time fluorescence measurement or electrochemical end-point detection (steps 5 and 6). 
Methodology of sequence-specific magnetocapture of SARS-CoV-2 RdRp plasmid followed by detection using qLAMP

Next, we investigated two types of sequence-specific magnetocapture assay - direct (Scheme 1) and indirect magnetocapture (Scheme 2) of the clinically relevant $10^{2}-10^{3}$ copies of plasmid from $40 \mu \mathrm{L}$ aqueous solution. For direct sequence-specific magnetocapture, a 5'biotinylated probe oligonucleotide (having $\mathrm{a} b$ sequence) was first immobilized on the streptavidin-coated magnetic beads. This was followed by blocking and then annealing with target nucleic acid carrying the $R d R p$ gene (containing a $\mathrm{b}^{*}$ sequence complementary to probe $\mathrm{b}$, Scheme 1). In the indirect sequence-specific magnetocapture, the 5'-biotinylated probe oligonucleotides were first annealed with target nucleic acid (to form the plasmid-probe binary complex in the solution) followed by immobilization on streptavidin-coated magnetic beads (Scheme 2). In both cases, magnetic decantation wash (and resulting physical separation) was then utilized to rid of any polymerase inhibitors or non-target nucleic acid from the magnetic bead-bound binary complex. The direct and indirect magneto-extraction were completed with assay time 60 and 30 min, respectively. Next, the magnetic bead carrying the plasmid-probe binary complex was subjected to in situ (on-bead) qLAMP to assess the effectiveness of capture (Figure 1A). In both cases, the no template control (NTC) assays were carried out with direct or indirect sequencespecific magnetocapture of a blank sample (i.e., without target), magnetic decantation, and then qLAMP. Additionally, internal target controls comprising of pure (i.e., without magnetocapture) $10^{3}$ copies of $R d R p$ plasmid were included in each assay.

For direct sequence-specific magnetocapture (Figure 1B, Figure S3A), 100 and 1000 copies of plasmids were detected at the cycle threshold value $\left(\mathrm{C}_{t}\right)$ of $51 \pm 1.4$ and $43.1 \pm 2.7$, 
respectively $\left(C_{t}\right.$ value of target control having 1000 copies was at $\left.13.8 \pm 4.9\right)$. In comparison, indirect sequence-specific magneto-extraction followed by qLAMP detected 100 and 1000 copies at $\mathrm{C}_{\mathrm{t}}$ values of $25.3 \pm 12.1$ and $18.1 \pm 5.3$, respectively (Figure $1 \mathrm{~B}$, Figure $\mathrm{S} 3 \mathrm{~B}$ ). Due to the statistically significant difference (using two-tailed Student's t-test) in the $C_{t}$ values for both 100 and 1000 copies between the direct and indirect sequence-specific magnetocapture strategies, the latter was used for the further assays. The indirect sequence-specific magneto-enrichment probably utilized the superior diffusion rate of the smaller biotinylated probe oligonucleotide (compared to the bulkier magnetic bead immobilized probe) in finding and annealing with the target nucleic acid. The indirect capture method has also demonstrated superior target binding for nucleic acid capture as well as antigen-antibody interaction in prior studies ${ }^{28,47}$. It also validates another independent observation that the bead-immobilized complementary probe (similar to direct sequence-specific magnetocapture) took over $2 \mathrm{~h}$ to capture more than $70 \%$ target nucleic acid from solution ${ }^{48}$. Additionally, indirect sequence-specific magnetocapture does not require blocking, and therefore was faster, inexpensive, and has reduced pipetting steps. Despite these advantages and demonstrated proof-of-concept of the indirect sequence-specific magnetocaptureamplification assay, it risks non-specific carryover contamination from the components of complex biofluids and host nucleic acid. In the subsequent experiments (below), we, therefore, explored whether the stated advantage of indirect sequence-specific magnetocapture could be successfully employed to sequence-specifically detect nucleic acid targets from human genomic DNA (hgDNA)-spiked and serum-spiked aqueous solution. 


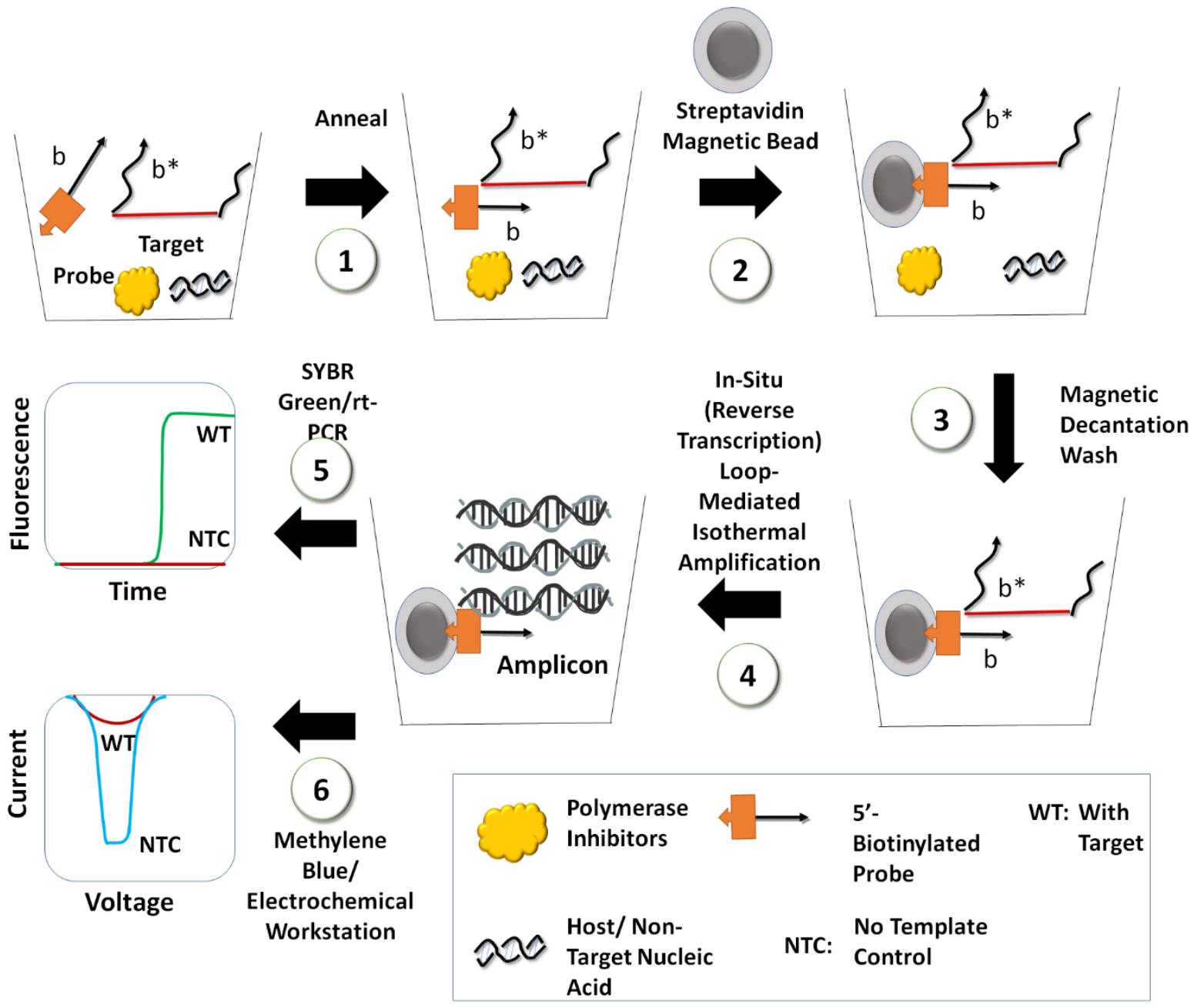

Scheme 2. Indirect sequence-specific magnetocapture of target nucleic acid (present with host nucleic acid and polymerase inhibitors from complex biofluid) by annealing the biotinylated probe and target (step 1), followed by immobilization with the magnetic beads (step 2). Following magnetic decantation wash (step 3), the target bound magnetic beads was used for in situ LAMP (or reverse transcriptase LAMP) amplification (step 4). This was detected by quantitative real-time fluorescence measurement (step 5) or electrochemical end-point detection (step 6).

\section{Detection of SARS-CoV-2 RdRp plasmid DNA from hgDNA- and serum-spiked samples}

In clinical samples, the target nucleic acid may often remain mixed in matrices such as sputum, swab, or serum containing various polymerase inhibitors that may hinder downstream real-time PCR application ${ }^{49}$. The presence of hgDNA may sometimes interfere in the efficiency as 
well as specificity of downstream amplification. Serum, on the other hand, is also a component of

A.

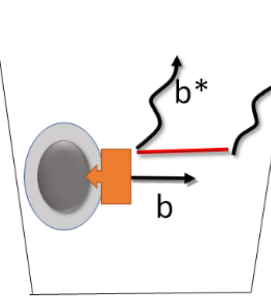

Magnetocapture-

Extracted Nucleic

Acid

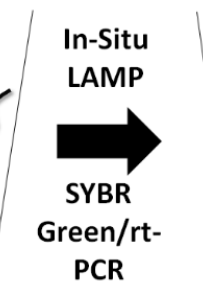

Instrument
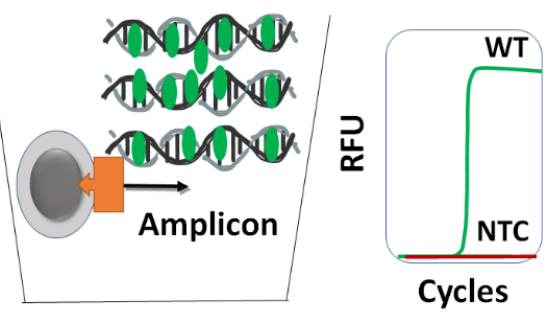

NTC: No Template Control

WT: With Target

SYBR Green I

B.

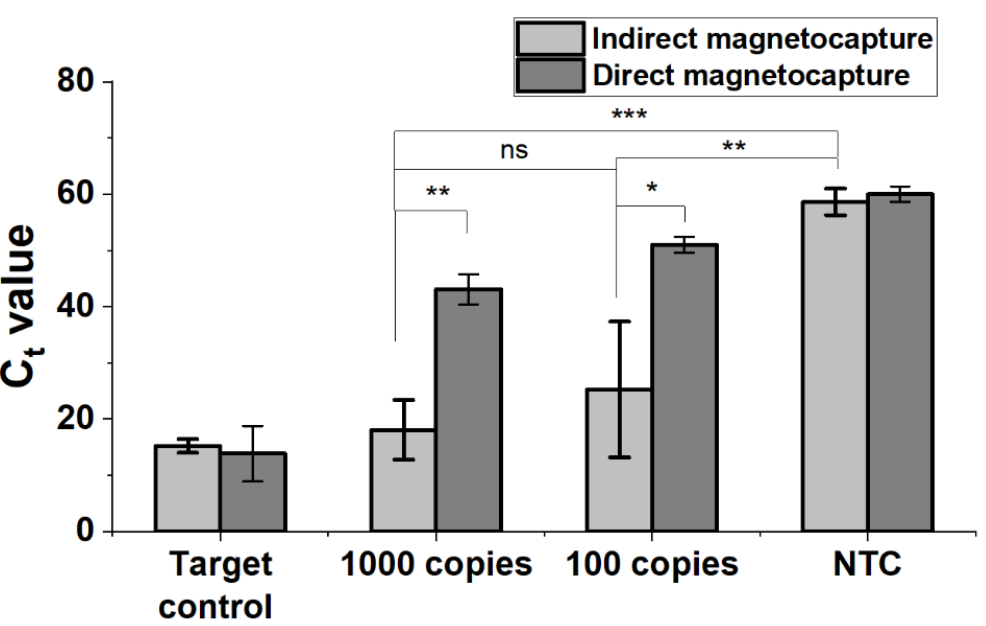

Figure 1. Comparison of direct and indirect sequence-specific magnetocapture followed by in situ qLAMP for the detection of 100 - 1000 copies of SARS-CoV-2 plasmid. A, Scheme of in situ qLAMP with magnetocaptured 100 and 1000 copies of SARS-CoV-2 RdRp plasmid DNA from aqueous solution. B, Comparison of cycle threshold $\left(\mathrm{C}_{\mathrm{t}}\right)$ values for direct vs indirect magnetocapture of 100 and 1000 copies of SARS-CoV-2 RdRp plasmid DNA followed by in situ qLAMP. Target control LAMP experiments were performed with $10^{3}$ copies of pure plasmid DNA (without any magnetocapture). NTC assays comprised of magnetocapture experiments that were carried out without any target nucleic acid followed by qLAMP. Error bars represent standard deviation $(\mathrm{n}=3)$. $* \mathrm{P} \leq 0.05, * * \mathrm{P} \leq 0.01, * * * \mathrm{P} \leq$ $0.001, * * * * \mathrm{P} \leq 0.0001$

viral transport media (VTM) and has polymerase inhibitory property ${ }^{50}$. Therefore, magnetopreconcentration and amplification of the target nucleic acid from samples spiked with excess host nucleic acid or those containing inhibiting complex biofluid such as serum would simulate the assay performance in a real-life scenario. To investigate the effectiveness of indirect sequence- 
specific magneto-preconcentration integrated with situ amplification assay, $100-1000$ copies of the target $R d R p$ plasmid ( $1-10$ fg, respectively) in $40 \mu \mathrm{L}$ was spiked with excess hgDNA ( $1 \mathrm{ng}$ ) in aqueous solution or 10\% fetal bovine serum (FBS) samples (Scheme 2). Accordingly, NTC experiments also included indirect sequence-specific magneto-preconcentration involving $R d R p$ plasmid-free samples containing either hgDNA or serum. After incubating with the biotinylated probes, the annealed target-biotinylated probe complex was then captured with streptavidin-coated MNP followed by in situ qLAMP (Figure 2A). As expected, the pure plasmid DNA (positive control) had the lowest $C_{t}$ values. This was followed by a slightly higher magnitude of $C_{t}$ values for $10^{2}$ and $10^{3}$ copies for hgDNA spiked target sample (Figure 2B, Figure S4A, and C) and serum spiked sample (Figure 2C, Figure S4B and D). The relatively higher $C_{t}$ values for serum spiked samples were probably due to the continued presence of minute quantity polymerase inhibitor proteins from serum (i.e., carryover contamination). These experiments demonstrated the proofof-concept magneto-preconcentration detection of clinically relevant 100 and 1000 copies of double-stranded DNA in $40 \mu \mathrm{L}$ (i.e., the limit of detection or LoD of 2.5 copies $/ \mu \mathrm{L}$ or $4.1 \mathrm{aM}$ ) from hgDNA spiked and serum spiked sample. Additionally, the same-to-answer turnaround time for the enrichment-amplification assay was $1.5-2 \mathrm{~h}$. 
A.

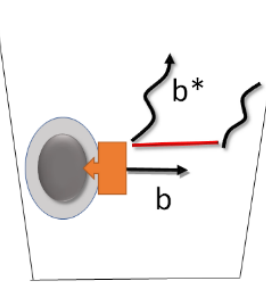

Magnetocapture-

Extracted Nucleic

Acid

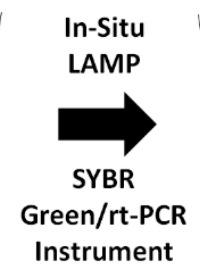

Instrument

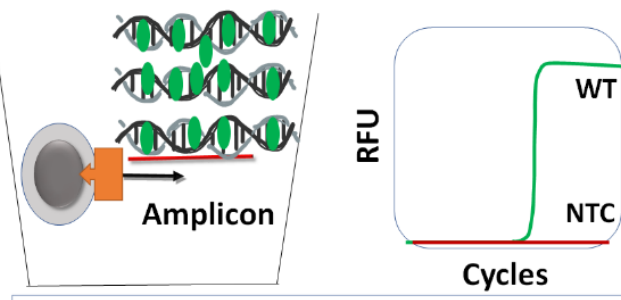

NTC: No Template Control WT: With Target SYBR Green I

B.

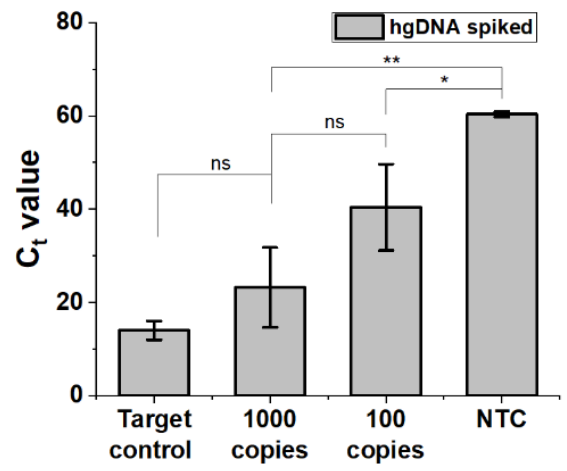

C.

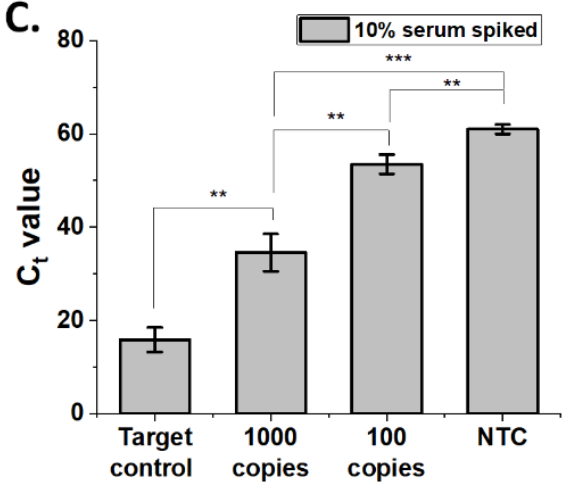

Figure 2. Indirect sequence-specific magnetocapture of 100 and 1000 copies of SARS-CoV-2 RdRp plasmid DNA from aqueous solution spiked with hgDNA or serum $(10 \%$, v/v) followed by in situ qLAMP. A, Scheme of in situ qLAMP with magnetocaptured 100 and 1000 copies of SARS-CoV-2 RdRp plasmid DNA. B, Indirect magnetocapture of 100 and 1000 copies ( $1-10 \mathrm{fg}$ ) of SARS-CoV-2 RdRp plasmid DNA from aqueous solution spiked with hgDNA ( $1 \mathrm{ng}$ ) followed by in situ qLAMP. C, Indirect magnetocapture of 100 and 1000 copies of SARS-CoV-2 RdRp plasmid DNA from aqueous solution spiked with serum $(10 \%$, v/v) followed by in situ qLAMP. Target control LAMP experiments were performed with $10^{3}$ copies of pure plasmid DNA (without any magnetocapture). NTC assays comprised of magnetocapture experiments that were carried out without any target nucleic acid followed by qLAMP. Error bars represent standard deviation $(\mathrm{n}=3) .{ }^{*} \mathrm{P} \leq 0.05,{ }^{* *} \mathrm{P} \leq 0.01,{ }^{* * *} \mathrm{P} \leq 0.001,{ }^{* * * *} \mathrm{P} \leq 0.0001$.

\section{Detection of SARS-CoV-2 RdRp RNA spiked with hgDNA and serum}

Next, we investigated whether the magnetic bead-mediated sequence-specific preconcentration followed by in situ amplification could be utilized for detecting clinically relevant concentrations of SARS-CoV-2 RdRp RNA (in vitro transcribed from plasmid). The in 
vitro transcription, characterization, and quantification of RNA containing $R d R p$, standard curve generation for quantitative real-time reverse transcription LAMP (qRT-LAMP) assay (using pure RNA) have been described in the Supporting Information Section 6 and Figure S5. Like RdRp plasmid DNA, the qRT-LAMP involving pure $R d R p$ RNA was able to sense 10 copies/reaction with four orders of dynamic range (Figure S6). The detection of the in vitro transcribed RNA carrying the $R d R p$ gene of SARS-CoV-2 was then investigated for indirect magneto-enrichment followed by one step amplification procedure. Like indirect sequence-specific capture of $R d R p$ plasmid DNA, 100 - 1000 copies of RNA present in $40 \mu \mathrm{L}$ aqueous media, hgDNA spiked aqueous solution, or serum-spiked sample was annealed with the 5'-biotinylated probe oligonucleotide. The solution was then incubated with streptavidin magnetic beads for immobilization on the latter. Following washing (for removal of inhibitor and non-target nucleic acid), the beads were then subjected to in situ qRT-LAMP (Figure 3A). Similar to plasmid DNA experiments, the NTC experiments included indirect sequence-specific magneto-enrichment involving target RNA-free aqueous samples or solutions containing either hgDNA or serum.

For the magneto-enrichment of RNA from the aqueous sample, 100 and 1000 copies of target RNA were detected with a $\mathrm{C}_{\mathrm{t}}$ of $45.1 \pm 8.1,32.4 \pm 4.1$, respectively (Figure 3B). Besides, internal target control qRT-LAMP containing pure (i.e., without magneto-extraction) $10^{3}$ copies of $R d R p$ RNA were detected with a $C_{\mathrm{t}}$ value $9.3 \pm 2.3$ while the $\mathrm{NTC} \mathrm{C}_{\mathrm{t}}$ was higher than 60 (Figure 3B, Supporting Information Figure S7A and C). For the RNA present in an aqueous solution containing excess hgDNA, 100 and 1000 copies of RNA were detected with slightly higher (compared to aqueous magneto-enrichment) $\mathrm{C}_{\mathrm{t}}$ of $42 \pm 2.8$ and $36.2 \pm 6.2$, respectively (Figure 3C, Supporting Information Figure S7B and D). This result suggests that proposed magnetoenrichment and follow-up in situ qRT-LAMP amplification would be possible in presence of 
hgDNA, therefore bypassing the need for TaqMan probe for downstream readouts. The slightly higher $\mathrm{C}_{t}$ value for detection from hgDNA spiked samples (compared to magnetocapture of RNA from aqueous media, above) reflected a similar trend as found for plasmid DNA magnetocapture. This could be a result of lesser effectiveness in target capture specificity due to the presence of excess $\left(10^{5}\right.$ times higher in weight) of hgDNA. Similar to plasmid DNA detection, the experiments, therefore, validated that the assay could effectively detect clinically relevant quantity $(100-1000$ copies in $40 \mu \mathrm{L}$ or $4.1-41 \mathrm{aM})$ SARS-CoV-2 RdRp RNA from aqueous and hgDNA spiked samples within $1.5-2 \mathrm{~h}$.

Next, we investigated the detection of RNA present in serum-spiked (5\%, v/v) sample. This assay was of high relevance as the $0.5-5 \%$ serum spiked aqueous sample resembled the composition of the nasopharyngeal swabs sample transporting VTM used in SARS-CoV-2 diagnosis $^{51,52}$. The assay efficacy and turnaround time would thus be predictive if the magnetocapture were to be used in actual clinical sample detection. In this assay, however, we initially detected little or no amplification or melt curve signature from the magnetocapture of RNA from serum spiked solution (Figure S8). In a literature search, we found that RNA has a halflife of as little as $15 \mathrm{sec}$ in serum due to possible RNase mediated degradation ${ }^{53}$. As a result, amplification using SARS-CoV-2 RNA from saliva or swab samples (i.e., without any extraction) has inadequate analytical sensitivity and could be $26-2000$ fold lower compared to that for a preextracted RNA ${ }^{23}$. Additionally, the analytical sensitivity of an SYBR I-based qRT-PCR without any RNA extraction was significantly lower than its TaqMan probe-enabled counterpart ${ }^{23}$. However, both the analytical sensitivity and specificity could be improved by the addition of EDTA or RNase inhibitor for RNase activity mitigation ${ }^{23}$. Accordingly, we found that prior addition of RNAase inhibitor and $25 \mathrm{mM}$ EDTA with serum improved the RNA stability in our 
assay as well. With the stated optimization and same magnetocapture method as described above, we assessed the efficacy of the magnetocapture-amplification to detect 100 and 1000 copies of RNA in a 40 uL RNA-EDTA-serum mixture (Figure 3D). The $C_{t}$ values were relatively higher (56.2 \pm 7.7 and $47.3 \pm 2.2$ for 100 and 1000 copies, respectively) compared to RNA detection from aqueous media or hgDNA spiked sample. This was probably due to continued degradation of some target RNA and would cause a slightly longer sample-to-answer turnaround time ( $2.0 \mathrm{~h}$, Figure 3D, Figure S9). Nevertheless, our method was able to detect SARS-CoV-2 RdRp RNA with clinically relevant sensitivity of 100 copies/reaction ( 2.5 copies $/ \mu \mathrm{L}$ or $4.1 \mathrm{aM})$ from aqueous and hgDNA-spiked samples and 1000 copies/reaction (25 copies/ $\mu \mathrm{L}$ or $41 \mathrm{aM})$ from serum-spiked samples. 
A.

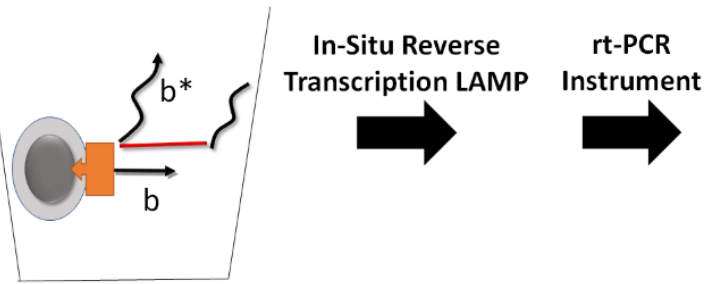

Magnetocapture-

Extracted Nucleic

Acid
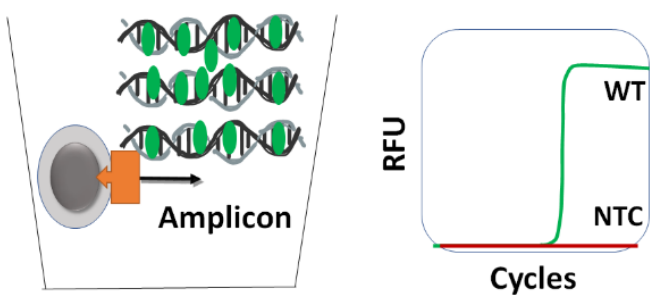

NTC: No Template Control WT: With Target SYBR Green I
B.

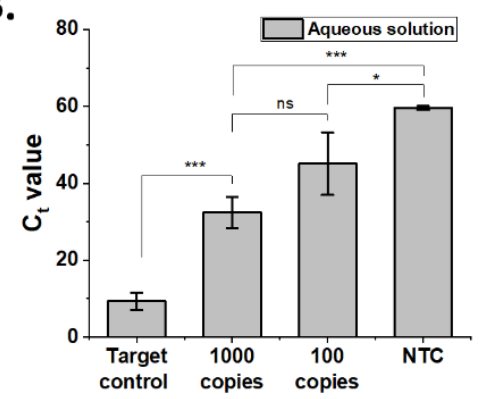

C.

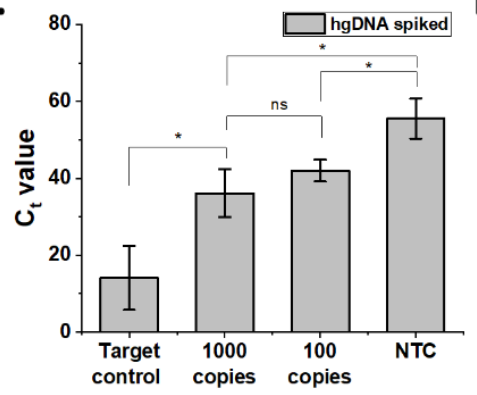

D.

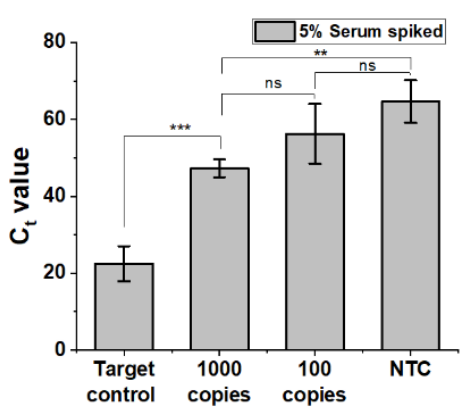

Figure 3. Indirect sequence-specific magnetocapture of 100 and 1000 copies of in vitro transcribed SARS-CoV-2 $R d R p$ RNA from aqueous media, or aqueous sample spiked with hgDNA (1 ng), or serum (5\%, v/v) followed by in situ qRT-LAMP. A, Scheme of in situ qRT-LAMP with magnetocaptured 100 and 1000 copies of SARS-CoV-2 RdRp RNA. B, Indirect magnetocapture of 100 and 1000 copies of SARS-CoV-2 $R d R p$ RNA from aqueous solution followed by in situ qRT-LAMP. C, Indirect magnetocapture of 100 and 1000 copies of SARS-CoV-2 RdRp RNA from aqueous solution spiked with hgDNA ( $1 \mathrm{ng}$ ) followed by in situ qRT-LAMP. D, Indirect magnetocapture of 100 and 1000 copies of SARS-CoV-2 RdRp RNA from aqueous solution spiked serum (5\%, v/v) followed by in situ qRTLAMP. Target control qRT-LAMP experiments were performed with $10^{3}$ copies of $R d R p$ RNA (without any magnetocapture). NTC assays comprised of magnetocapture experiments that were carried out without any target RNA followed by qRT-LAMP Error bars represent standard deviation $(\mathrm{n}=3) .{ }^{*} \mathrm{P} \leq 0.05,{ }^{*} \mathrm{P} \leq 0.01, * * * \mathrm{P} \leq 0.001$, $* * * * \mathrm{P} \leq 0.0001$. 


\section{Detection of SARS-CoV-2 plasmid DNA and RNA using sequence-specific indirect magneto- extraction and electrochemical LAMP}

Next, we examined the detection of $100-1000$ copies of $R d R p$ plasmid DNA or RNA using the magneto-extraction integrated LAMP with end-point electrochemical readout. Electrochemical detection of NAATs is advantageous as it facilitates nucleic acid-sensing without bulky instruments like real-time PCR. However, the performance of such electrochemical NAAT sensing systems should also match their gold standard fluorescence-based real-time PCR instrument counterparts. With this objective, we investigated whether the proposed sequencespecific indirect magneto-extraction is amenable to downstream electrochemical end-point LAMP (eLAMP) or electrochemical reverse transcription end-point LAMP (eRT-LAMP) readout on a commercial screen-printed electrode. The methylene blue reagent was utilized as the electron mediator due to its superiority over other electrochemical NAAT redox mediators such as sodium molybdate or osmium tetroxide ${ }^{54}$. Similarly, square wave voltammetry (SWV) was applied due to its proven greater sensitivity thanks to minimal capacitive as well as background currents. In principle, the presence of an amplicon would prevent the methylene blue-mediated electron transfer to the electrode, thereby reducing the current signal (Figure 4A). Additionally, the presence of increasingly greater amount of template copies in the reaction would generate a higher amount of amplicon. This would trap more methylene blue and cause successively lesser current transfer to the electrode, generating gradually lower signal in the process. The same results could alternatively be observed through increasing magnitude of the signal \% change $\left(S(I)=\left(I_{0}-I_{1}\right) x\right.$ $100 / I_{0}$ ), where $I_{0}$ and $I_{1}$ were the current signals generated from samples corresponding to the NTC and target containing samples, respectively), representing the relative change in the current signal with respect to the NTC samples. 
Accordingly, eLAMP or eRT-LAMP assay involving pure (i.e., without magnetocapture) $10^{1}-10^{4}$ copies of DNA and RNA showed a gradually decreasing peak current signal that was progressively lower than the negative template control or NTC (Figure 4B and C). The comparison of peak current signal with NTC was thus utilized to assess the detectability of the target nucleic acid. Similarly, the signal \% change also demonstrated a gradual increase in S(I) with increasing concentrations (Figure S10). In the magneto-extraction assisted amplification as well, the eLAMP current signal for 100 - 1000 copies was compared with NTC assays performed with a target nucleic acid-free magneto-extraction. A current signal difference of $100-1000$ copies of nucleic acid compared to NTC would therefore reflect the effectiveness of the sensing approach. Besides NTC, an internal target control (TC) comprising of an eLAMP (or eRT-LAMP as relevant) with pure (i.e., without magnetocapture) $10^{3}$ copies of plasmid or RNA (as applicable) was included in each assay.

The significant difference in peak current signal for magneto-extraction of target plasmid from aqueous, hgDNA spiked, and serum spiked sample validated the compatibility of magnetoextraction with eLAMP (Figure 5B and C). The peak current signal difference between NTC and $100-1000$ copies of plasmid reproducibly remained in the range of $15-25 \mu \mathrm{A}$ (approximately $30-50 \%$ in terms of signal \% change, Figure S11A). Although the detection of 100 copies of plasmid was expected to produce a higher current signal than 1000 copies, interestingly, the signal difference between the detection of magneto-extracted 100 and 1000 copies themselves was not statistically significant. Similarly for the $R d R p$ RNA magneto-extraction-amplification, a lesser, albeit still statistically significant, current difference $(7-10 \mu \mathrm{A})$ was observed compared to their respective NTCs. This lesser difference in current compared to NTC (12 - 30\% in terms of signal \% change, Figure S11B) was probably due to partial RNA degradation, resulting in a lesser 
amplicon generation. It led to a higher quantity of free methylene blue mediator in solution, causing reduced current difference with that of NTC. Similar to plasmid DNA magneto-extraction, RNA detection could also not differentiate between 100 - 1000 copies of magneto-extracted RNA. While the experiments thus validated the magneto-extraction amplification assays for both plasmid DNA and RNA, it could not distinguish between magnetocaptured 100 - 1000 copies of nucleic

A.

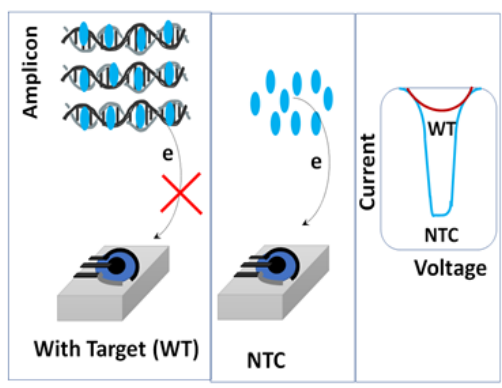

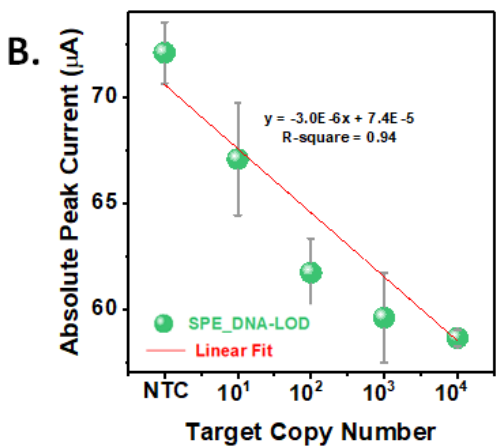

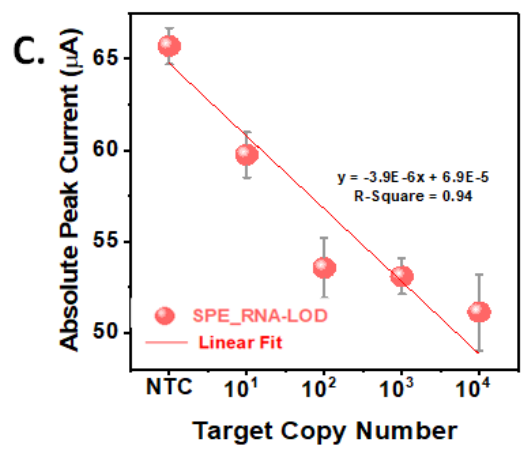

Figure 4. Electrochemical LAMP studies conducted on pure $10^{1}-10^{4}$ copies of nucleic acid (without magnetocapture). A, Mechanism of amplicon-mediated methylene blue sequestration and subsequent reduction of current. B, LoD for eLAMP on pure $10^{1}-10^{4}$ copies SARS-CoV-2 RdRp plasmid DNA/reaction. C, LoD for eRT-LAMP on pure $10^{1}-10^{4}$ copies SARS-CoV-2 $R d R p$ RNA. NTC assays comprised of eLAMP or eRT-LAMP without any template nucleic acid addition.

acid, an aspect to be improved in future developments (please see below for rationalization). The assay performance for rescuing and detecting target nucleic acid from aqueous, hgDNA spiked, and serum spiked samples was also equivalent to those using real-time fluorescence readout, with both providing detection for at least 2.5 copies $/ \mu \mathrm{L}$ targets. Overall, the magneto-enrichment followed by eLAMP thus successfully demonstrated the detection of clinically relevant concentration (2.5 copies $/ \mu \mathrm{L}$ or $4.1 \mathrm{aM})$ of target SARS-CoV-2 nucleic acid with a sample-toanswer turnaround time of $2 \mathrm{~h}$. 


\section{Comparison of LAMP using a pure nucleic acid template and magneto-extraction assisted}

\section{LAMP}

An amplification experiment such as quantitative real-time PCR (qPCR) or quantitative real-time LAMP (qLAMP) displays remarkable analytical sensitivity ( 1 - 10 copies/reaction) when it utilizes pure pre-extracted nucleic acid as a template. However, its performance is contingent on the purity of the nucleic acid and could get affected by the presence of polymerase inhibitors. Additionally, the presence of excess host nucleic acid may also cause non-specific background signal, requiring sequence-specific probes such as TaqMan. The proposed indirect sequence-specific magneto-enrichment assay, on the other hand, preconcentrates the target nucleic acid and relies on succeeding in situ LAMP step to detect the analyte with high analytical sensitivity. In this section, we have explored a comparison between qLAMP and eLAMP assays using the pure nucleic acid template and also weighed their performance against the indirect sequence-specific magneto-extraction followed by LAMP (qLAMP or eLAMP). This was carried out in the context of detecting 100 copies of target nucleic acid. The readings from LAMP assays carried out in this study using pure nucleic acid template i.e., $\mathrm{C}_{\mathrm{t}}$ values from qLAMP in Figure $\mathrm{S} 2$, qRT-LAMP in Figure S6, absolute current signals from eLAMP or eRT-LAMP assays in Figure 4B and $\mathrm{C}$ were utilized for this purpose. These were compared with corresponding $\mathrm{C}_{\mathrm{t}}$ values or current signals obtained from indirect sequence-specific magneto-enrichment assisted LAMP experiments (Figure 1B, Figure 2, Figure 3 and 5). It should be noted that in the qLAMP assays, cycle threshold values would approximately correspond to signal detection time since the fluorescence measurement was performed at the end of every $1 \mathrm{~min}$ cycle. The turnaround time 
for qLAMP assays was calculated based on the presumption that the $\mathrm{C}_{\mathrm{t}}$ value could be considered as the time for signal visualization and accordingly decision making.

In the context of analytical sensitivity with a pure nucleic acid template, the qLAMP (or qRT-LAMP) assays had a similar performance as that of eLAMP (or eRT-LAMP) with both being able to sense 10 copies/reaction (Figures S2, S6, and Figure 4). However, qLAMP (or qRT-LAMP) was advantageous in terms of assay turnaround time as it relies on real-time signal growth measurement and not on end-point measurement as in eLAMP (or eRT-LAMP). Similarly, magneto-enrichment-assisted qLAMP (or qRT-LAMP) displayed equivalent LoD (2.5 copies/ $\mu \mathrm{L}$, except for RNA detection from serum-spiked sample, showing LoD of 25 copies $/ \mu \mathrm{L}$ ) as that of magnetocapture-eLAMP (or eRT-LAMP) (Figures 1 - 5). The assay turnaround time for magnetoextraction assisted qLAMP or eLAMP, also, would not be significantly different from each other as both required the 25 - 30 min magneto-enrichment followed by an almost $80 \mathrm{~min}$ (60 min of LAMP followed by 20 min inactivation) amplification step. Despite similar analytical sensitivity and turnaround time, a workflow comprising of indirect sequence-specific magneto-enrichment assisted eLAMP (or eRT-LAMP) utilizing a portable electrochemical workstation would be especially suitable in resource-constrained settings, thanks to its independence of centralized instruments.

In terms of assay turnaround time, a qLAMP (or qRT-LAMP) experiment using a pure nucleic acid template would take approximately 1.2 - $1.5 \mathrm{~h}$ for detecting 100 copies $(40-60 \mathrm{~min}$ for nucleic acid extraction ${ }^{55}$, followed by at least 20 - 30 min for LAMP signal detection). In comparison, the proposed magneto-extraction-assisted qLAMP would require a sample-to-answer turnaround time of $1-1.5 \mathrm{~h}(25-30 \mathrm{~min}$ for magneto-extraction and $35-55 \mathrm{~min}$ of LAMP readout) to detect 100 copies. It may appear that the $C_{t}$ values for magneto-extraction-assisted 
LAMP are significantly higher (and even outside the range of qLAMP standard curves shown in Figure S2 and Figure S6) than those using qLAMP using the pure nucleic acid template. However, it must also be considered that magneto-extraction-assisted in situ $20 \mu \mathrm{L}$ qLAMP assays are using only $1 / 5^{\text {th }}$ of the magnetic beads $(2 \mu \mathrm{L})$ being used for magneto-extraction. This was due to the observation that adding more ( $>2 \mu \mathrm{L}$ ) beads in a $20 \mu \mathrm{L}$ reaction would inhibit the qLAMP (data not shown). In situ amplification of the entire $10 \mu \mathrm{L}$ bead from the amgnetocapture therefore would necessitate proportionately increasing reaction volume to $100 \mu \mathrm{L}$. Therefore, we anticipate that the $\mathrm{C}_{\mathrm{t}}$ value (and accordingly assay time) of the magneto-extraction-assisted qLAMP would substantially reduce if a higher reaction volume containing a greater amount of magnetic beads were to be used, although this would increase the assay cost (please see below for an estimate). Overall, the qLAMP using the pure nucleic acid template and the proposed indirect sequencespecific magneto-extraction assisted qLAMP detected clinically relevant 100 copies of target nucleic acid with similar turnaround time, despite the latter being a minimally instrument-intensive technique.

In contrast, indirect sequence-specific magneto-extraction-assisted eLAMP for detecting 100 copies of target nucleic acid had reduced assay timing compared to the eLAMP assay using 100 copies of pure nucleic acid as the template. Unlike the qLAMP experiments that provided a real-time signal visualization, one needed to run the entire $80 \mathrm{~min}$ (60 min of assay followed by 20 min of inactivation) eLAMP assay before recording the SWV end-point measurements (5 min per sample). Therefore, the eLAMP using pure nucleic acid as template experiments had a turnaround time of $120-140 \mathrm{~min}$ (40 - 60 min of nucleic acid extraction, 80 min of LAMP, and 5 min of SWV). In comparison, the indirect sequence-specific magneto-extraction assisted LAMP 
was shorter in duration and could be completed in 90 - $120 \mathrm{~min}$ (30 min of magneto-extraction, 80 min of LAMP, and 5 min of SWV).

Another interesting observation was that the eLAMP (or eRT-LAMP) end-point current signal was not significantly different between 100 and 1000 copies of magneto-extracted target nucleic acids. While this observation may seem unusual, it should be noted that the $C_{t}$ values for magneto-enrichment-assisted qLAMP on 100 and 1000 copies were also not significantly different (Figure $1-3$ ). In addition, unlike the real-time cycle threshold measurement in qLAMP, the eLAMP quantifies the current at the end of the reaction. Its measurement would therefore be proportional to the total amount of amplicon presence in the reaction, which could be evaluated using the final fluorescence readings of each of these assays. An assessment of the representative amplification curves thus revealed that the final fluorescence (at the $60^{\text {th }}$ cycle) was in the order of $800-1000$ RFU (Figures S3B, S4A and B, S7A and B), and thus might rationalize this observation. Furthermore, the current signal during the eLAMP or eRT-LAMP performed on the $10^{2}$ and $10^{3}$ copies of pure nucleic acid, while significantly differentiable from NTC, was not highly distinguishable from each other (Figure 4B and C). These factors, either individually, or in combination with a homemade master mix may have led to a non-distinguishable current signal between magneto-extracted 100 and 1000 copies of target nucleic acid. While this still facilitates a clear "yes/no" decision-making concerning the presence of the analyte, it would (in our opinion) be a potential limitation of the magnetocapture-eLAMP assays due to their inability to determine the viral copy number. Overall, the indirect sequence-specific magnetocapture followed by eLAMP (or eRT-LAMP) on 100 copies of the target required a lower sample-to-answer turnaround time than eLAMP (or eRT-LAMP) assays involving using pure 100 copies of the nucleic acid template. 
A.

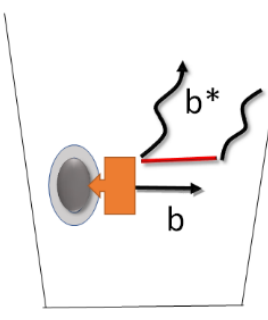

Magnetocapture-

Extracted Nucleic

Acid

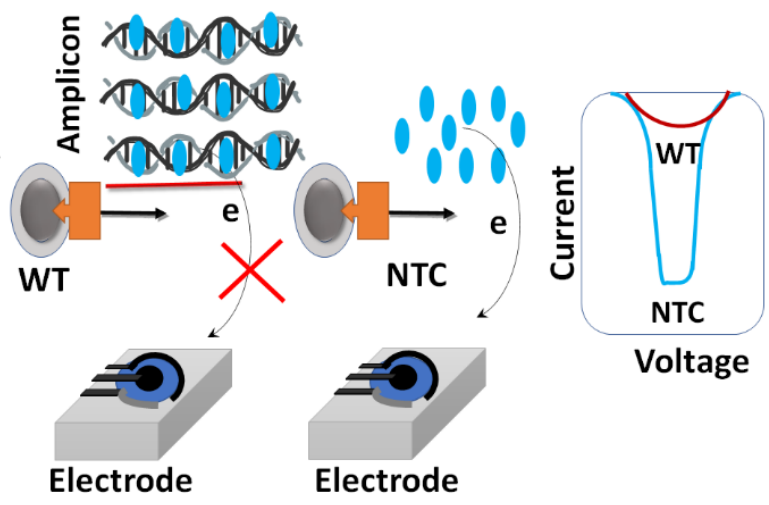

WT: With Target

NTC: No Template Control

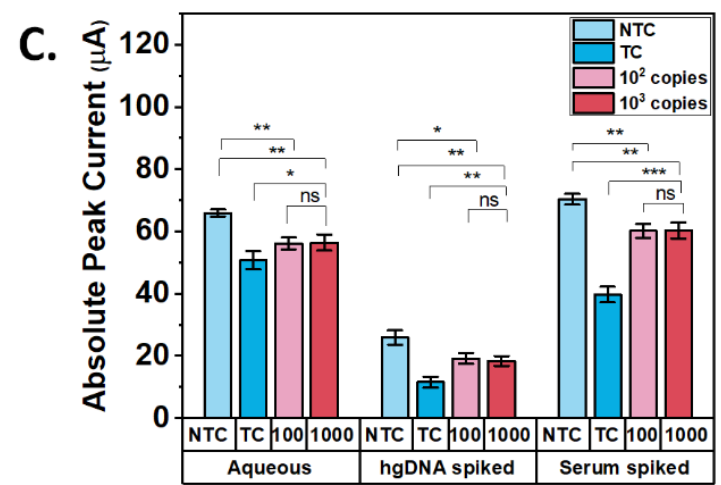

Figure 5. Indirect sequence-specific magnetocapture of 100 and 1000 copies of SARS-CoV-2 RdRp plasmid DNA and RNA from aqueous media, or aqueous sample spiked with hgDNA (1 ng), or serum followed by in situ electrochemical end-point (reverse transcription) LAMP (eLAMP or eRT-LAMP). A, Scheme of in situ eLAMP with magnetocaptured 100 and 1000 copies of SARS-CoV-2 RdRp plasmid DNA or RNA. B, Indirect magnetocapture of 100 and 1000 copies of SARS-CoV-2 RdRp plasmid DNA from aqueous solution, or aqueous sample spiked with hgDNA ( $1 \mathrm{ng})$, or serum (10\%, v/v) followed by in situ eLAMP. C, Indirect magnetocapture of 100 and 1000 copies of SARS-CoV-2 RdRp RNA from aqueous solution, or aqueous sample spiked with hgDNA (1 ng), or serum (5\%, v/v) followed by in situ eRT-LAMP. Target control (TC) eLAMP or eRT-LAMP experiments were performed with $10^{3}$ copies of $R d R p$ DNA or RNA, respectively (without any magnetocapture). NTC assays comprised of magnetocapture experiments that were carried out without any target DNA or RNA followed by eLAMP or eRTLAMP, respectively. Error bars represent standard deviation $(\mathrm{n}=3) . * \mathrm{P} \leq 0.05, * * \mathrm{P} \leq 0.01, * * * \mathrm{P} \leq 0.001, * * * * \mathrm{P} \leq$ 0.0001 


\section{Discussion, cost, limitation of the assay, and future studies}

In this work, we have addressed the primary limitations of existing NAAT and iNAAT methods; (i) need for thermal cycling (for qRT-PCR), (ii) requirement of prior RNA extraction (for all NAATs), (iii) necessity of TaqMan-like sequence-specific probes to circumvent nonspecific background signal (for qRT-PCR) ${ }^{56}$, and utilization of real-time PCR machines restricted to centralized labs (for all NAATs). All these factors increase the assay cost, centralize the test, and enhance turnaround time. The spin-column or magnetic bead-based RNA detection kits (inclusive of extraction and amplification module) would cost $\$ 6-11 /$ sample with turnaround time (sample-to-answer) ranging from $4 \mathrm{~h}$ to $1 \mathrm{day}^{23,57}$. While automated cartridges integrate the whole sample-to-answer in a single continuous workflow, they are also costlier (over $\$ 10$ per assay $)^{58}$.

In our work, we have instead adopted an indirect sequence-specific magnetocapture assay that has been designed to be user-friendly, less instrument-intensive, and inexpensive. This assay could be executed with simply a benchtop heater (or magnetic stirrer or water bath reaching $65^{\circ} \mathrm{C}$ ) and a magnet with a turnaround time of 30 min. Choosing LAMP for downstream detection purposes also avoided the need for thermal cycling. The follow-up LAMP was performed in situ, not requiring elution of target nucleic acid from the magnetic bead, and therefore saving time in the process. The downstream LAMP showed compatibility with both real-time fluorescence as well as electrochemical readout, and therefore was suitable for both centralized as well as resourcelimited field applications. The extraction of nucleic acid using magnetocapture and then detection of amplicons using a portable electrochemical workstation makes it easier to administer the detection in a resource-limited setting. Indeed, certain electrochemical techniques such as SWV is 
highly sensitive, quantitative, cost-effective, and compatible with multiplexing ${ }^{59}$. The magnetoextraction followed by NAAT can thus be easily combined with portability and sensitivity of electrochemical workstations for field-based molecular diagnosis at limited resources settings such as community centers near an outbreak.

The proposed assay successfully demonstrated proof-of-concept detection of $100-1000$ copies (equivalent to 2.5 - 25 copies/ $\mu \mathrm{L}$ ) SARS-CoV-2 RdRp plasmid DNA and RNA from aqueous, hgDNA spiked, and serum spiked VTM-simulating samples. In terms of analytical sensitivity, its performance has been equivalent or superior to other published magnetocapture assisted extraction assays listed in Table S1. Although the magnetocapture-qLAMP (or qRTLAMP) assay was able to successfully detect $100-1000$ copies in a short time and differentiate the same from NTC, it was unable to significantly distinguish the signal between the 100 and 1000 copies in all cases. The low signal difference between 100 and 1000 copies may have originated due to the use of in-house qLAMP mix and possibly be improved for commercial LAMP mastermix. Additionally, a different primer set with even higher specificity could also help in detecting and differentiating close copy numbers of viral nucleic acid. Similarly, the nondifferentiation of current signal between 100 - 1000 copies along with a clear distinction compared to NTC was observed for the magnetocapture-eLAMP or eRT-LAMP assays as well. As discussed earlier, the similar current signal of $100-1000$ copies probably originated from the end-point measurement that considered the total amplicon quantity and might be differentiated if the eLAMP was run for a shorter time ${ }^{60}$. The turnaround time (sample-to-answer) for both fluorescence and electrochemical workflows was $1.5-2.5 \mathrm{~h}$, showing the potential of near-point-of-care detection.

When assessed for cost benefits, the raw materials involved in our method costs INR 224 or \$3.04 per assay (involving all commercial reagents, Supporting Information Table S4), implying 
that our integrated magnetocapture amplification would not be costlier than existing RNA extraction and qRT-PCR kits despite the faster and limited-resource-friendly detection. With inhouse prepared magnetic beads and enzymes, the assay cost could be expected to go down even further. Given the general nature of detection and low assay cost, this method would also be able to sense any target pathogen nucleic acid and is therefore expected to see broader applications in the future. We are currently optimizing the assay with clinical samples and lateral flow assaybased detection to create a visual readout. Although not explored here, the sequence-specific magnetic enrichment followed by LAMP could in principle be integrated with a microfluidic setup as well and will be taken up in future studies.

\section{Conclusion}

This work addressed several critical issues currently concerning the NAAT molecular diagnosis, namely, the requirement of RNA extraction to remove polymerase inhibitors, use of TaqMan probe for specificity, thermal cycling, and requirement of centralized real-time PCR machine. While isothermal amplifications such as LAMP partially address some of these challenges, their optimal performance is dependent on the presence of pure nucleic acid as a template. Therefore, LAMP using the pure nucleic acid template cannot overcome the need of an instrument-intensive nucleic acid extraction step in the overall workflow. It also does not address the need for sequence-recognizing reporter molecular beacon-like probes for specificity.

In this study, we developed and demonstrated the superiority of an indirect sequencespecific magneto-preconcentration assay of target nucleic acid over that of a direct sequencespecific magneto-preconcentration. Utilizing downstream real-time SYBR-based fluorescence readout and electrochemical end-point LAMP, this study demonstrated proof-of-concept detection 
of clinically relevant concentration $(2.5-25$ copies/ $\mu \mathrm{L}$ and $4.1-41 \mathrm{aM})$ SARS-CoV-2 $R d R p$ gene-bearing plasmid DNA and RNA. The assay successfully detected the aforementioned concentrations of target nucleic acids from an aqueous sample as well as real-life simulating hgDNA spiked and VTM-mimic samples. The analytical sensitivity of the assay at 2.5 copies $/ \mu \mathrm{L}$ is comparable to other published as well as commercial assays for SARS-CoV-2 detection ${ }^{61}$ (Table S1). This work is presumably the first study demonstrating an electrochemical LAMP (eLAMP) involving pure SARS-CoV-2 nucleic acid. It is also novel in proving the superiority of indirect sequence-specific magneto-preconcentration (followed by in situ LAMP) over direct magnetopreconcentration in nucleic acid NAAT biosensing, and the first to do so with LAMP as the downstream NAAT. In addition, the developed assay would probably be the first to demonstrate its effectiveness in magneto-extracting and then detecting SARS-CoV-2 nucleic acid mixed with real life-simulating sample involving the field application-friendly electrochemical readout.

This study is probably the first to evaluate the role of hgDNA and serum-based polymerase inhibitors on sequence-specific indirect magneto-extraction and downstream NAAT. It also compared magnetic preconcentration assisted electrochemical LAMP with that of a magnetocapture combined with a real-time fluorescence LAMP. In the process, it conclusively demonstrated their equivalent analytical sensitivity that in turn enhanced the operational flexibility of the overall workflow. Furthermore, it quantitatively compared (for the first time in published literature, to the best of our knowledge) the sequence-specific magneto-extraction-LAMP assay with that of LAMP using the pure nucleic acid template in terms of turnaround time and assay cost. In doing so, it demonstrated that the former has a similar or superior performance than the latter in detecting clinically relevant 100 copies of target nucleic acid. The study is general in nature and could be extended to detect other pathogens as well. Similarly, the indirect sequence- 
specific magnetic enrichment could also be integrated with other NAATs. Overall, our assay provided a sensitive, low-cost, near-point-of-care, sample-to-answer, and non-instrumentintensive method for detecting pathogen nucleic acid.

\section{Materials and Methods}

Materials. Escherichia coli str. K-12 substr. MG1655 plasmid construct with RNA dependent RNA polymerase ( $R d R p)$ gene with T7 RNA polymerase promoter (4538 bp) was purchased from Addgene (plasmid \#14567, https://www.addgene.org/145671/). The Bst 2.0 polymerase, RTx enzyme, dNTP, and SnaBI were procured from NEB, USA. The SYBR I $(10,000 \mathrm{X}$ concentrated) was purchased from Invitrogen, USA. Molecular biology grade water was purchased from HiMedia, India. The RNase inhibitor was purchased from Takara. Streptavidincoated magnetic beads were purchased from Sigma-Aldrich (\# 11641778001) or Invitrogen (Dynabeads M-280). 5'-biotinylated probe having the b sequence (5'-[BIO]-AAA AAA AAA ACG AGC AAG AAC AAG TGA GGC CAT AAT TC, HPLC purified) was purchased from Sigma-Aldrich. Primer oligonucleotides (desalting purified) were purchased from Eurofin or Sigma-Aldrich, India.

LAMP reaction and primer optimization using real-time fluorescence readout. A realtime quantitative LAMP (qLAMP) experiment was performed on $10^{3}$ copies of ORF1ab containing plasmid with three sets of primers. The final LAMP reaction $(20 \mu 1)$ contained the three primer pairs in the following final concentrations: $0.2 \mu \mathrm{M}$ outer primers, and $1.6 \mu \mathrm{M}$ forward and backward inner primers, $0.8 \mu \mathrm{M}$ forward and back loop primers (for primer sets 1 and 3) ${ }^{19,62}$. For primer set 2, $0.4 \mu \mathrm{M}$ outer primers, $0.332 \mu \mathrm{M}$ forward and backward inner primer, $1 \mu \mathrm{M}$ forward loop primers, and $0.4 \mu \mathrm{M}$ back loop primers were utilized in final concentration ${ }^{46}$. The reaction 
mixture also contained $2.0 \mu \mathrm{L}$ of $10 \times$ Bst 2.0 DNA polymerase reaction buffer $[1 \times$ containing 20 $\mathrm{mM}$ Tris- $\mathrm{HCl}, 50 \mathrm{mM} \mathrm{KCl}, 10 \mathrm{mM}\left(\mathrm{NH}_{4}\right)_{2} \mathrm{SO}_{4}, 2 \mathrm{mM} \mathrm{MgSO}_{4}, 0.1 \%$ Tween-20, pH 8.8], $1.4 \mathrm{mM}$ dNTPs, $2.0 \mu \mathrm{L}$ SYBR I (final concentration $1 \mathrm{X}$ diluted from 10,000X stock), $0.5 \mu \mathrm{L}$ of an $8 \mathrm{U} \mu \mathrm{l}$ concentration of Bst 2.0 DNA polymerase, $6 \mathrm{mM} \mathrm{MgSO}_{4}$ and $1 \mu 1$ template (alternatively, $2 \mu \mathrm{L}$ magnetic bead for magnetocapture assays). For quantitative real-time reverse transcription LAMP (qRT-LAMP), 7U $(0.25 \mu \mathrm{L})$ of reverse transcriptase RTx (NEB) was additionally added to the above. The qLAMP (or qRT-LAMP) reaction was set at the following settings for each cycle with a fluorescence monitoring step, $65^{\circ} \mathrm{C}$ for 1 minute for primer set $1,64^{\circ} \mathrm{C}$ for 1 minute for primer set $2,60^{\circ} \mathrm{C}$ for 1 minute for primer set 3 followed by thermal melting analysis step. The cycles were repeated 60 times (unless otherwise stated) in a CFX Maestro or CFX Connect real-time PCR (rt-PCR) machine (BioRad).

Direct sequence-specific magnetocapture followed by in situ LAMP. $0.1 \mu \mathrm{M} 5^{\prime}$ biotinylated probe oligonucleotide (having a $\mathrm{b}$ sequence) was first immobilized on the $10-\mu \mathrm{g}$ streptavidin-coated magnetic nanoparticles by 20 minutes incubation. After incubation with washbinding buffer ( $5 \mathrm{mM}$ Tris-HCl, $0.5 \mathrm{mM}$ EDTA, $1 \mathrm{M} \mathrm{NaCl}$, pH 7.5) the biotinylated probe bound streptavidin-coated MNP was blocked with 1\% BSA for 20 minutes. After three times of washing with wash-binding buffer, the probe-MNP complex was incubated (15 min, using vortex enabled mild shaking) with snap-cooled $\left(65^{\circ} \mathrm{C} 5 \mathrm{~min}\right.$ followed by cooling in ice for $\left.5 \mathrm{~min}\right) 100$ or 1000 copies of target plasmid carrying the RdRp gene (containing a b* sequence) in $40 \mu \mathrm{L}$. For the negative control (NTC), water is used instead of plasmid during the magnetocapture. After three successive washes using magnetic decantation with $200 \mu \mathrm{L}$ wash-binding buffer, the target nucleic acid bound MNP was resuspended in $10 \mu \mathrm{L}$ nuclease-free water. $2 \mu \mathrm{L}$ beads are used for $20 \mu \mathrm{L}$ in situ qLAMP reactions using primer set 2 as described above. 
Indirect sequence-specific magnetocapture followed by in situ LAMP. In this method, $0.1 \mu \mathrm{M}$ 5'-biotinylated probe oligonucleotides having b sequence was first annealed with 100 or 1000 copies of target plasmid or $R d R p$ RNA copies in $40 \mu \mathrm{L}$ by heating at $65^{\circ} \mathrm{C}$ ( 2 minutes), followed by incubation in ice $(5 \mathrm{~min})$, and then $15 \mathrm{~min}$ benchtop incubation at room temperature. For magnetocapture from hgDNA spiked sample, the plasmid or RNA was instead present in 40 $\mu \mathrm{L}$ solution containing $1 \mathrm{ng}$ MCF-7 extracted hgDNA and then subjected to magnetocapture as described. Similarly, for serum spiked samples, the plasmid or RNA was instead present in $40 \mu \mathrm{L}$ solution containing $10 \%(\mathrm{v} / \mathrm{v}$, for DNA) or $5 \%(\mathrm{v} / \mathrm{v}$, for RNA) serum and then subjected to magnetocapture as described (please see below for the protocol of RNA magnetocapture from RNase and EDTA treated serum). This step generates the plasmid (or RNA)-probe binary complex which is then followed by 15 min incubation (using vortex enabled mild shaking) with $10 \mu \mathrm{g}$ streptavidin-coated MNP at room temperature. For the negative control (NTC), the magnetocapture was performed with the same solution but lacking the target nucleic acid. After three times washes with wash-binding buffer using magnetic decantation the target nucleic acid bound MNP has resuspended in $10 \mu \mathrm{l}$ nuclease-free water. $2 \mu \mathrm{L}$ beads were used for $20 \mu \mathrm{L}$ in situ qLAMP reaction using primer set 2 as described above.

Plasmid digestion \& in vitro transcription. $1 \mu \mathrm{g}$ target plasmid was linearized with $1 \mu \mathrm{L}$ SnaBI enzyme $(10 \mathrm{U} / \mu \mathrm{L})$ and $1 \mathrm{X}$ NEB CutSmart buffer $(50 \mathrm{mM}$ Potassium Acetate, $20 \mathrm{mM}$ Trisacetate, $10 \mathrm{mM}$ Magnesium Acetate, $100 \mu \mathrm{g} / \mathrm{ml} \mathrm{BSA} \mathrm{pH} 7.9$ at $25^{\circ} \mathrm{C}$ ) by incubating at $37^{\circ} \mathrm{C}$ for 1 hour (final volume $50 \mu \mathrm{L}$ ). Next, the digestion was stopped by an additional enzyme inactivation step $\left(80^{\circ} \mathrm{C}\right.$ for $\left.20 \mathrm{mins}\right)$. Next, $4 \mu \mathrm{L}$ of the digested sample was loaded into $2 \%$ agarose gel to check the linearized product. $1 \mu \mathrm{g}$ of digested plasmid was then added with $1 \mathrm{X}$ NTP buffer mix (NEB), $2 \mu \mathrm{L}$ of T7 RNA polymerase in $20 \mu \mathrm{L}$ reaction, and incubated at $37^{\circ} \mathrm{C}$ for 2 hours. The 
resultant RNA product was cleaned with Monarch ${ }^{\circledR}$ RNA Cleanup Kit (NEB) as per the manufacturer's instruction. Briefly, $100 \mu \mathrm{L}$ of RNA clean-up binding buffer was added to the 50 $\mu \mathrm{L}$ sample obtained from the reaction. $150 \mu \mathrm{L}$ of pure ethanol is added to the mixture and loaded into the column provided in the kit. After centrifugation at $13000 \mathrm{rpm}$ for 1 minute, the column is again washed with $500 \mu \mathrm{L}$ wash buffer and finally, the product was eluted in $50 \mu \mathrm{L}$ nuclease-free water.

RNA quantification by qPCR. cDNA was synthesized using the PrimeScript 1 st strand cDNA Synthesis Kit (Takara) as per the manufacturer's instruction. Briefly, $1 \mu \mathrm{g}$ RNA was mixed with $0.4 \mu \mathrm{L}$ random hexamers, $1 \mathrm{mM}$ dNTP mixture and snap cooled $\left(65^{\circ} \mathrm{C}\right.$ for 5 minutes followed by incubation in ice for 5 minutes, final volume $10 \mu \mathrm{L}$ ). Then, cDNA was synthesized with $1 \mathrm{X}$ prime script buffer, $100 \mathrm{U}$ of reverse transcriptase, and template RNA primer mixture and was incubated at $30^{\circ} \mathrm{C}$ for 10 minutes followed by $50^{\circ} \mathrm{C}$ for 30 minutes (final volume $20 \mu \mathrm{L}$ total). Parallelly a standard curve for copy numbers vs $\mathrm{C}_{\mathrm{t}}$ value was generated by qPCR on plasmid DNA. The qPCR was performed to determine the cDNA concentration and analyzed using a CFX Maestro (BioRad) with SYBR Green Real-Time PCR Master Mix Plus (HiMedia) followed by thermal melting analysis. The primers were designed against the $R d R p$ gene of the plasmid (Eurofins, please see sequences in Supporting Information Table S3). The cDNA was diluted 1/400 fold and subjected to qPCR to determine its concentration.

\section{Magnetocapture of 100 and 1000 copies of in vitro transcribed RNA from $5 \%$ serum}

spiked solution. $6.6 \%$ fetal bovine serum (Gibco), $1.0 \mu \mathrm{L}$ of RNase inhibitor, $25 \mathrm{mM}$ EDTA (final) are incubated together at $37^{\circ} \mathrm{C}$ for $1 \mathrm{~h}$ to a final volume of $30 \mu \mathrm{L} .100$ and 1000 copies of RNA in aqueous solution (final volume $10 \mu \mathrm{L}$ ) was heated up to $65^{\circ} \mathrm{C}$ for 2 minutes followed by immediate addition of preincubated EDTA-serum mixture (above) and $0.1 \mu \mathrm{M}$ (final 
concentration) of 5'-biotinylated probe oligonucleotides (sequence b) to a final volume of $40 \mu \mathrm{L}$ and then cooled in ice. After incubation of $15 \mathrm{~min}$ at room temperature, the mixture was then immobilized on streptavidin magnetic beads as discussed in the plasmid immobilization on beads. For the negative control (NTC), the magnetocapture was performed with the same solution but lacking the target nucleic acid. After washing as described above, the beads were resuspended in $10 \mu \mathrm{L}$ nuclease-free water and $2 \mu \mathrm{L}$ of that was subjected to in situ qRT-LAMP as described above.

Electrochemical LAMP assays. For eLAMP or eRT-LAMP using the pure nucleic acid template, the assay was performed on $10^{1}-10^{5}$ copies of nucleic acid ( $R d R p$ plasmid DNA or $R d R p$ RNA)/ $25 \mu \mathrm{L}$ of reaction. For magnetocapture followed by eLAMP or eRT-LAMP assays, 2 $\mu \mathrm{L}$ magnetic beads containing immobilized target nucleic acid was added to $25 \mu \mathrm{L}$ electrochemical LAMP reaction having a composition as described below. A $25 \mu \mathrm{L}$ eLAMP or eRT-LAMP reaction comprised of $2.5 \mu \mathrm{L}$ of $10 \times$ Bst 2.0 DNA polymerase reaction buffer [ $1 \times$ containing 20 mM Tris- $\mathrm{HCl}, 50 \mathrm{mM} \mathrm{KCl}, 10 \mathrm{mM}\left(\mathrm{NH}_{4}\right)_{2} \mathrm{SO}_{4}, 2$ mM MgSO $4,0.1 \%$ Tween-20, pH 8.8], $1.4 \mathrm{mM}$ dNTPs, $0.4 \mu \mathrm{M}$ outer primers, $0.332 \mu \mathrm{M}$ forward and backward inner primer, $1 \mu \mathrm{M}$ forward loop primers, $0.4 \mu \mathrm{M}$ back loop primers (primer set 2), $0.5 \mu \mathrm{L}$ Bst 2.0 polymerase, and $50 \mu \mathrm{M}$ methylene blue. During magnetocapture followed by eLAMP or eRT-LAMP, 1000 copies of nonmagnetocaptured plasmid DNA or RNA was used as the positive control. For eRT-LAMP, 7 U of reverse transcriptase (RTx) was also used for $25 \mu \mathrm{L}$ of reaction. The assays were set at the following settings for each cycle, $64^{\circ} \mathrm{C}$ for 1 minute for 60 cycles followed by heat inactivation at $80^{\circ} \mathrm{C}$ for 20 minutes in a thermal cycler (Eppendorf). The resultant LAMP amplicons were electrochemically analyzed.

Electrochemical measurement. The samples were tested electrochemically using commercially purchased screen-printed electrode (SPE) with carbon, carbon, and silver as 
working, counter, and quasi-reference electrodes, respectively. The electroanalytical study was performed via square-wave voltammetry (SWV) using Metrohm Autolab PGSTAT302N potentiostat/galvanostat. The voltage was swept from a range of 0 to $-1 \mathrm{~V}$ for 5 consecutive cycles and the current was recorded simultaneously. All the electroanalytical data presented are averaged over 4 cycles, excluding the first cycle since an intense current was observed in the first cycle due to electric double layer (EDL) charging of the electrode. The percent signal change (S(I)) was calculated using this formula,

$$
\mathrm{S}(\mathrm{I})=\frac{\mathrm{I}_{0}-\mathrm{I}_{1}}{\mathrm{I}_{0}} * 100 \%
$$

Where $I_{0}$ and $I_{1}$ were the current signals generated from samples corresponding to the NTC and target containing samples, respectively.

\section{Acknowledgment}

The authors are thankful to Mission on Nano Science and Technology, Department of Science and Technology, Government of India for Nanomission grant (ref no DST/NM/NB/2018/189) and Department of Biotechnology grant (ref no BT/PR40297/COD/139/6/2020). They are also thankful to the Bennett University seed grant awarded to Dr. Ashvani Kumar, and Bennett University Ph.D. scholarship for Mr. Sayantan Tripathy and Ms. Tanvi Agarkar. The authors are grateful to Prof. Rajinder S. Chauhan for constant scholarly support. 


\section{Conflict of Interest}

The authors have filed Indian patents no 202111028722 and 202111037358 as part of this work.

\section{Author Contributions}

S.T., T.A., A.T., M.S., A.K., and S.G. envisioned the study. S.T. and S.G. designed, conducted, and analyzed the nucleic acid magnetocapture, in vitro transcription, and NAAT experiments. T.A. and A.K. designed, conducted, and analyzed the electrochemical measurements. M.S. guided plasmid procurement, microbiology, cell culture, and in vitro transcription. A.T. guided clinical perspectives of molecular diagnosis and requirements in limited-resource settings. S.T., T.A., M.S., A.K., and S.G. wrote the paper. All authors edited and approved the final manuscript.

\section{Supporting Information}

The supporting information section contains a comprehensive literature search on magneto-extraction assisted LAMP methods, additional comments on experimental methods and materials, LAMP primer sequences and optimization, LAMP LoD on plasmid and RNA, representative LAMP amplification profiles and melt curve analysis, RT-PCR primer sequences and standard curve, in vitro transcription and RNA quantification, electrochemical LAMP presented in the form of signal \% change, and cost analysis. 


\section{REFERENCES}

(1) Wu, F.; Zhao, S.; Yu, B.; Chen, Y.-M.; Wang, W.; Song, Z.-G.; Hu, Y.; Tao, Z.-W.; Tian, J.-H.; Pei, Y.Y.; Yuan, M.-L.; Zhang, Y.-L.; Dai, F.-H.; Liu, Y.; Wang, Q.-M.; Zheng, J.-J.; Xu, L.; Holmes, E. C.; Zhang, Y.-Z. A New Coronavirus Associated with Human Respiratory Disease in China. Nature 2020, 579 (7798), 265-269. https://doi.org/10.1038/s41586-020-2008-3.

(2) Chen, Z.; Boon, S. S.; Wang, M. H.; Chan, R. W. Y.; Chan, P. K. S. Genomic and Evolutionary Comparison between SARS-CoV-2 and Other Human Coronaviruses. J. Virol. Methods 2021, 289, 114032. https://doi.org/10.1016/j.jviromet.2020.114032.

(3) Shannon, A.; Le, N. T.-T.; Selisko, B.; Eydoux, C.; Alvarez, K.; Guillemot, J.-C.; Decroly, E.; Peersen, O.; Ferron, F.; Canard, B. Remdesivir and SARS-CoV-2: Structural Requirements at Both Nsp12 RdRp and Nsp14 Exonuclease Active-Sites. Antiviral Res. 2020, 178, 104793. https://doi.org/10.1016/j.antiviral.2020.104793.

(4) Robson, F.; Khan, K. S.; Le, T. K.; Paris, C.; Demirbag, S.; Barfuss, P.; Rocchi, P.; Ng, W.-L. Coronavirus RNA Proofreading: Molecular Basis and Therapeutic Targeting. Mol. Cell 2020, 79 (5), 710-727. https://doi.org/10.1016/j.molcel.2020.07.027.

(5) Pruijssers, A. J.; Denison, M. R. Nucleoside Analogues for the Treatment of Coronavirus Infections. Curr. Opin. Virol. 2019, 35, 57-62. https://doi.org/10.1016/j.coviro.2019.04.002.

(6) Johns Hopkins Coronavirus Resource Center: Home https://coronavirus.jhu.edu/ (accessed 2022 $01-24)$.

(7) Zhou, L.; Ayeh, S. K.; Chidambaram, V.; Karakousis, P. C. Modes of Transmission of SARS-CoV-2 and Evidence for Preventive Behavioral Interventions. BMC Infect. Dis. 2021, 21 (1), 496. https://doi.org/10.1186/s12879-021-06222-4.

(8) Mabey, D.; Peeling, R. The Optimal Features of a Rapid Point-of-Care Diagnostic Test. In Revolutionizing Tropical Medicine; John Wiley \& Sons, Ltd, 2019; pp 81-87. https://doi.org/10.1002/9781119282686.ch3.

(9) Diagnostics, Q. FDA Instructions on Detectimg SARS-CoV-2 RNA with Real-Time RT-PCR. FDA.

(10) Uwamino, Y.; Wakui, M.; Aoki, W.; Kurafuji, T.; Yanagita, E.; Morita, M.; Nagata, M.; Inose, R.; Noguchi, M.; Yokota, H.; Hasegawa, N.; Saya, H.; Murata, M.; Keio Donner Project Team. Evaluation of the Usability of Various Rapid Antibody Tests in the Diagnostic Application for COVID-19. Ann. Clin. Biochem. 2021, 58 (3), 174-180. https://doi.org/10.1177/0004563220984827.

(11) Jegerlehner, S.; Suter-Riniker, F.; Jent, P.; Bittel, P.; Nagler, M. Diagnostic Accuracy of a SARS-CoV2 Rapid Antigen Test in Real-Life Clinical Settings. Int. J. Infect. Dis. IJID Off. Publ. Int. Soc. Infect. Dis. 2021, 109, 118-122. https://doi.org/10.1016/j.ijid.2021.07.010.

(12) Peto, T.; Affron, D.; Afrough, B.; Agasu, A.; Ainsworth, M.; Allanson, A.; Allen, K.; Allen, C.; Archer, L.; Ashbridge, N.; Aurfan, I.; Avery, M.; Badenoch, E.; Bagga, P.; Balaji, R.; Baldwin, E.;

Barraclough, S.; Beane, C.; Bell, J.; Benford, T.; Bird, S.; Bishop, M.; Bloss, A.; Body, R.; Boulton, R.; Bown, A.; Bratten, C.; Bridgeman, C.; Britton, D.; Brooks, T.; Broughton-Smith, M.; Brown, P.; Buck, B.; Butcher, E.; Byrne, W.; Calderon, G.; Campbell, S.; Carr, O.; Carter, P.; Carter, D.; Cathrall, M.; Catton, M.; Chadwick, J.; Chapman, D.; Chau, K. K.; Chaudary, T.; Chidavaenzi, S.; Chilcott, S.; Choi, B.; Claasen, H.; Clark, S.; Clarke, R.; Clarke, D.; Clayton, R.; Collins, K.; Colston, R.; Connolly, J.; Cook, E.; Corcoran, M.; Corley, B.; Costello, L.; Coulson, C.; Crook, A.; Crook, D. W.; D’Arcangelo, S.; Darby, M.-A.; Davis, J.; Koning, R. de; Derbyshire, P.; Devall, P.; Dolman, M.; Draper, N.; Driver, M.; Dyas, S.; Eaton, E.; Edwards, J.; Elderfield, R.; Ellis, K.; Ellis, G.; Elwell, S.; Evans, R.; Evans, B.; Evans, M.; Evans, R.; Eyre, D.; Fahey, C.; Fenech, V.; Field, J.; Field, A.; Foord, T.; Fowler, T.; French, M.; Fuchs, H.; Gan, J.; Gernon, J.; Ghadiali, G.; Ghuman, N.; Gibbons, K.; 
Gill, G.; Gilmour, K.; Goel, A.; Gordon, S.; Graham, T.; Grassam-Rowe, A.; Green, D.; Gronert, A.; Gumsley-Read, T.; Hall, C.; Hallis, B.; Hammond, S.; Hammond, P.; Hanney, B.; Hardy, V.; Harker, G.; Harris, A.; Havinden-Williams, M.; Hazell, E.; Henry, J.; Hicklin, K.; Hollier, K.; Holloway, B.; Hoosdally, S. J.; Hopkins, S.; Hughes, L.; Hurdowar, S.; Hurford, S.-A.; Jackman, J.; Jackson, H.; Johns, R.; Johnston, S.; Jones, J.; Kanyowa, T.; Keating-Fedders, K.; Kempson, S.; Khan, I.; Khulusi, B.; Knight, T.; Krishna, A.; Lahert, P.; Lampshire, Z.; Lasserson, D.; Lee, K.; Lee, L. Y. W.; Legard, A.; Leggio, C.; Liu, J.; Lockett, T.; Logue, C.; Lucas, V.; Lumley, S. F.; Maripuri, V.; Markham, D.; Marshall, E.; Matthews, P. C.; Mckee, S.; McKee, D. F.; McLeod, N.; McNulty, A.; Mellor, F.; Michel, R.; Mighiu, A.; Miller, J.; Mirza, Z.; Mistry, H.; Mitchell, J.; Moeser, M. E.; Moore, S.; Muthuswamy, A.; Myers, D.; Nanson, G.; Newbury, M.; Nicol, S.; Nuttall, H.; Nwanaforo, J. J.; Oliver, L.; Osbourne, W.; Osbourne, J.; Otter, A.; Owen, J.; Panchalingam, S.; Papoulidis, D.; Pavon, J. D.; Peace, A.; Pearson, K.; Peck, L.; Pegg, A.; Pegler, S.; Permain, H.; Perumal, P.; Peto, L.; Peto, T. E. A.; Pham, T.; Pickford, H. L.; Pinkerton, M.; Platton, M.; Price, A.; Protheroe, E.; Purnell, H.; Rawden, L.; Read, S.; Reynard, C.; Ridge, S.; Ritter, T. G.; Robinson, J.; Robinson, P.; Rodger, G.; Rowe, C.; Rowell, B.; Rowlands, A.; Sampson, S.; Saunders, K.; Sayers, R.; Sears, J.; Sedgewick, R.; Seeney, L.; Selassie, A.; Shail, L.; Shallcross, J.; Sheppard, L.; Sherkat, A.; Siddiqui, S.; Sienkiewicz, A.; Sinha, L.; Smith, J.; Smith, E.; Stanton, E.; Starkey, T.; Stawiarski, A.; Sterry, A.; Stevens, J.; Stockbridge, M.; Stoesser, N.; Sukumaran, A.; Sweed, A.; Tatar, S.; Thomas, H.; Tibbins, C.; Tiley, S.; Timmins, J.; Tomas-Smith, C.; Topping, O.; Turek, E.; Neibler, T.; Trigg-Hogarth, K.; Truelove, E.; Turnbull, C.; Tyrrell, D.; Vaughan, A.; Vertannes, J.; Vipond, R.; Wagstaff, L.; Waldron, J.; Walker, P.; Walker, A. S.; Walters, M.; Wang, J. Y.; Watson, E.; Webberley, K.; Webster, K.; Westland, G.; Wickens, I.; Willcocks, J.; Willis, H.; Wilson, S.; Wilson, B.; Woodhead, L.; Wright, D.; Xavier, B.; Yelnoorkar, F.; Zeidan, L.; Zinyama, R. COVID-19: Rapid Antigen Detection for SARS-CoV-2 by Lateral Flow Assay: A National Systematic Evaluation of Sensitivity and Specificity for MassTesting. EClinicalMedicine 2021, 36. https://doi.org/10.1016/j.eclinm.2021.100924.

(13) CDC Diagnostic Tests for COVID-19.

(14) Wacker, M. J.; Godard, M. P. Analysis of One-Step and Two-Step Real-Time RT-PCR Using SuperScript III. J. Biomol. Tech. JBT 2005, 16 (3), 266-271.

(15) RT-PCR: One step vs. two step | IDT https://sg.idtdna.com/pages/support/faqs/what-are-theadvantages-and-disadvantages-of-one-step-vs.-two-step-rt-pcr (accessed 2021 -12 -04).

(16) Ganguli, A.; Mostafa, A.; Berger, J.; Aydin, M. Y.; Sun, F.; Ramirez, S. A. S. de; Valera, E.; Cunningham, B. T.; King, W. P.; Bashir, R. Rapid Isothermal Amplification and Portable Detection System for SARS-CoV-2. Proc. Natl. Acad. Sci. U. S. A. 2020, 117 (37), 22727-22735. https://doi.org/10.1073/pnas.2014739117.

(17) Notomi, T.; Okayama, H.; Masubuchi, H.; Yonekawa, T.; Watanabe, K.; Amino, N.; Hase, T. LoopMediated Isothermal Amplification of DNA. Nucleic Acids Res. 2000, 28 (12), e63.

(18) Dao Thi, V. L.; Herbst, K.; Boerner, K.; Meurer, M.; Kremer, L. P.; Kirrmaier, D.; Freistaedter, A.; Papagiannidis, D.; Galmozzi, C.; Stanifer, M. L.; Boulant, S.; Klein, S.; Chlanda, P.; Khalid, D.; Barreto Miranda, I.; Schnitzler, P.; Kräusslich, H.-G.; Knop, M.; Anders, S. A Colorimetric RT-LAMP Assay and LAMP-Sequencing for Detecting SARS-CoV-2 RNA in Clinical Samples. Sci. Transl. Med. 2020, 12 (556), eabc7075. https://doi.org/10.1126/scitranslmed.abc7075.

(19) El-Tholoth, M.; Bau, H. H.; Song, J. A Single and Two-Stage, Closed-Tube, Molecular Test for the 2019 Novel Coronavirus (COVID-19) at Home, Clinic, and Points of Entry. ChemRxiv Prepr. Serv. Chem. 2020. https://doi.org/10.26434/chemrxiv.11860137.

(20) Broughton, J. P.; Deng, X.; Yu, G.; Fasching, C. L.; Servellita, V.; Singh, J.; Miao, X.; Streithorst, J. A.; Granados, A.; Sotomayor-Gonzalez, A.; Zorn, K.; Gopez, A.; Hsu, E.; Gu, W.; Miller, S.; Pan, C.-Y.; Guevara, H.; Wadford, D. A.; Chen, J. S.; Chiu, C. Y. CRISPR-Cas12-Based Detection of SARS-CoV-2. Nat. Biotechnol. 2020, 38 (7), 870-874. https://doi.org/10.1038/s41587-020-0513-4. 
(21) Yu, L.; Wu, S.; Hao, X.; Dong, X.; Mao, L.; Pelechano, V.; Chen, W.-H.; Yin, X. Rapid Detection of COVID-19 Coronavirus Using a Reverse Transcriptional Loop-Mediated Isothermal Amplification (RT-LAMP) Diagnostic Platform. Clin. Chem. https://doi.org/10.1093/clinchem/hvaa102.

(22) Jayanath, N. Y.; Nguyen, L. T.; Vu, T. T.; Tran, L. D. Development of a Portable Electrochemical Loop Mediated Isothermal Amplification (LAMP) Device for Detection of Hepatitis B Virus. RSC Adv. 2018, 8 (61), 34954-34959. https://doi.org/10.1039/C8RA07235C.

(23) Pearson, J. D.; Trcka, D.; Lu, S.; Hyduk, S. J.; Jen, M.; Aynaud, M.-M.; Hernández, J. J.; Peidis, P.; Barrios-Rodiles, M.; Chan, K.; Woodgett, J.; Mazzulli, T.; Attisano, L.; Pelletier, L.; Cybulsky, M. I.; Wrana, J. L.; Bremner, R. Comparison of SARS-CoV-2 Indirect and Direct RT-QPCR Detection Methods. Virol. J. 2021, 18 (1), 99. https://doi.org/10.1186/s12985-021-01574-4.

(24) Oberacker, P.; Stepper, P.; Bond, D. M.; Höhn, S.; Focken, J.; Meyer, V.; Schelle, L.; Sugrue, V. J.; Jeunen, G.-J.; Moser, T.; Hore, S. R.; von Meyenn, F.; Hipp, K.; Hore, T. A.; Jurkowski, T. P. Bio-OnMagnetic-Beads (BOMB): Open Platform for High-Throughput Nucleic Acid Extraction and Manipulation. PLOS Biol. 2019, 17 (1), e3000107. https://doi.org/10.1371/journal.pbio.3000107.

(25) Hu, X.; Deng, Q.; Li, J.; Chen, J.; Wang, Z.; Zhang, X.; Fang, Z.; Li, H.; Zhao, Y.; Yu, P.; Li, W.; Wang, X.; Li, S.; Zhang, L.; Hou, T. Development and Clinical Application of a Rapid and Sensitive LoopMediated Isothermal Amplification Test for SARS-CoV-2 Infection. mSphere 2020, 5 (4). https://doi.org/10.1128/mSphere.00808-20.

(26) Wang, C.-H.; Lien, K.-Y.; Wang, T.-Y.; Chen, T.-Y.; Lee, G.-B. An Integrated Microfluidic LoopMediated-Isothermal-Amplification System for Rapid Sample Pre-Treatment and Detection of Viruses. Biosens. Bioelectron. 2011, 26 (5), 2045-2052. https://doi.org/10.1016/j.bios.2010.08.083.

(27) Zhang, L.; Wei, Q.; Han, Q.; Chen, Q.; Tai, W.; Zhang, J.; Song, Y.; Xia, X. Detection of Shigella in Milk and Clinical Samples by Magnetic Immunocaptured-Loop-Mediated Isothermal Amplification Assay. Front. Microbiol. 2018, 9. https://doi.org/10.3389/fmicb.2018.00094.

(28) Tsai, S. P.; Wong, A.; Mai, E.; Chan, P.; Mausisa, G.; Vasser, M.; Jhurani, P.; Jakobsen, M. H.; Wong, W. L. T.; Stephan, J.-P. Nucleic Acid Capture Assay, a New Method for Direct Quantitation of Nucleic Acids. Nucleic Acids Res. 2003, 31 (6), e25.

(29) Dynabeads M-280 Technical Bulletin.

(30) Wang, C.-H.; Lien, K.-Y.; Wu, J.-J.; Lee, G.-B. A Magnetic Bead-Based Assay for the Rapid Detection of Methicillin-Resistant Staphylococcus Aureus by Using a Microfluidic System with Integrated Loop-Mediated Isothermal Amplification. Lab. Chip 2011, 11 (8), 1521-1531. https://doi.org/10.1039/c0lc00430h.

(31) Lin, C.-L.; Chang, W.-H.; Wang, C.-H.; Lee, C.-H.; Chen, T.-Y.; Jan, F.-J.; Lee, G.-B. A Microfluidic System Integrated with Buried Optical Fibers for Detection of Phalaenopsis Orchid Pathogens. Biosens. Bioelectron. 2015, 63, 572-579. https://doi.org/10.1016/j.bios.2014.08.013.

(32) Chen, J.; Xu, X.; Huang, Z.; Luo, Y.; Tang, L.; Jiang, J.-H. BEAMing LAMP: Single-Molecule Capture and on-Bead Isothermal Amplification for Digital Detection of Hepatitis C Virus in Plasma. Chem. Commun. Camb. Engl. 2018, 54 (3), 291-294. https://doi.org/10.1039/c7cc08403j.

(33) Österdahl, M. F.; Lee, K. A.; Lochlainn, M. N.; Wilson, S.; Douthwaite, S.; Horsfall, R.; Sheedy, A.; Goldenberg, S. D.; Stanley, C. J.; Spector, T. D.; Steves, C. J. Detecting SARS-CoV-2 at Point of Care: Preliminary Data Comparing Loop-Mediated Isothermal Amplification (LAMP) to Polymerase Chain Reaction (PCR). BMC Infect. Dis. 2020, 20 (1), 783. https://doi.org/10.1186/s12879-02005484-8.

(34) Klein, S.; Müller, T. G.; Khalid, D.; Sonntag-Buck, V.; Heuser, A.-M.; Glass, B.; Meurer, M.; Morales, I.; Schillak, A.; Freistaedter, A.; Ambiel, I.; Winter, S. L.; Zimmermann, L.; Naumoska, T.; Bubeck, F.; Kirrmaier, D.; Ullrich, S.; Barreto Miranda, I.; Anders, S.; Grimm, D.; Schnitzler, P.; Knop, M.; Kräusslich, H.-G.; Dao Thi, V. L.; Börner, K.; Chlanda, P. SARS-CoV-2 RNA Extraction Using 
Magnetic Beads for Rapid Large-Scale Testing by RT-QPCR and RT-LAMP. Viruses 2020, 12 (8). https://doi.org/10.3390/v12080863.

(35) Yu, A. D.; Galatsis, K.; Zheng, J.; Le, J. Q.; Ma, D.; Perlman, S.; Rosbash, M. Development of a Saliva-Optimized RT-LAMP Assay for SARS-CoV-2. J. Biomol. Tech. JBT 2021, 32 (3), 102-113. https://doi.org/10.7171/jbt.21-3203-005.

(36) Bektaş, A.; Covington, M. F.; Aidelberg, G.; Arce, A.; Matute, T.; Núñez, I.; Walsh, J.; Boutboul, D.; Delaugerre, C.; Lindner, A. B.; Federici, F.; Jayaprakash, A. D. Accessible LAMP-Enabled Rapid Test (ALERT) for Detecting SARS-CoV-2. Viruses 2021, 13 (5). https://doi.org/10.3390/v13050742.

(37) Dignan, L. M.; Woolf, M. S.; Tomley, C. J.; Nauman, A. Q.; Landers, J. P. Multiplexed Centrifugal Microfluidic System for Dynamic Solid-Phase Purification of Polynucleic Acids Direct from Buccal Swabs. Anal. Chem. 2021, 93 (19), 7300-7309. https://doi.org/10.1021/acs.analchem.1c00842. Barclay, R. A.; Akhrymuk, I.; Patnaik, A.; Callahan, V.; Lehman, C.; Andersen, P.; Barbero, R.; Barksdale, S.; Dunlap, R.; Goldfarb, D.; Jones-Roe, T.; Kelly, R.; Kim, B.; Miao, S.; Munns, A.; Munns, D.; Patel, S.; Porter, E.; Ramsey, R.; Sahoo, S.; Swahn, O.; Warsh, J.; Kehn-Hall, K.; Lepene, B. Hydrogel Particles Improve Detection of SARS-CoV-2 RNA from Multiple Sample Types. Sci. Rep. 2020, 10 (1), 22425. https://doi.org/10.1038/s41598-020-78771-8.

(39) Chacón-Torres, J. C.; Reinoso, C.; Navas-León, D. G.; Briceño, S.; González, G. Optimized and Scalable Synthesis of Magnetic Nanoparticles for RNA Extraction in Response to Developing Countries' Needs in the Detection and Control of SARS-CoV-2. Sci. Rep. 2020, 10 (1), 19004. https://doi.org/10.1038/s41598-020-75798-9.

(40) Singh, V.; Batoo, K. M.; Singh, M. Fabrication of Chitosan-Coated Mixed Spinel Ferrite Integrated with Graphene Oxide (GO) for Magnetic Extraction of Viral RNA for Potential Detection of SARSCoV-2. Appl. Phys. Mater. Sci. Process. 2021, 127 (12), 960. https://doi.org/10.1007/s00339-02105067-7.

(41) Possebon, F. S.; Ullmann, L. S.; Malossi, C. D.; Miodutzki, G. T.; da Silva, E. C.; Machado, E. F.; Braga, I. S.; Pelaquim, I. F.; Araujo, J. P. J. A Fast and Cheap In-House Magnetic Bead RNA Extraction Method for COVID-19 Diagnosis. J. Virol. Methods 2022, 300, 114414. https://doi.org/10.1016/j.jviromet.2021.114414.

(42) Ramos-Mandujano, G.; Salunke, R.; Mfarrej, S.; Rachmadi, A. T.; Hala, S.; Xu, J.; Alofi, F. S.; Khogeer, A.; Hashem, A. M.; Almontashiri, N. A. M.; Alsomali, A.; Shinde, D. B.; Hamdan, S.; Hong, P.; Pain, A.; Li, M. A Robust, Safe, and Scalable Magnetic Nanoparticle Workflow for RNA Extraction of Pathogens from Clinical and Wastewater Samples (Global Challenges 4/2021). Glob. Chall. 2021, 5 (4), 2170041. https://doi.org/10.1002/gch2.202170041.

(43) Kang, J.; Li, Y.; Zhao, Y.; Wang, Y.; Ma, C.; Shi, C. Nucleic Acid Extraction without Electrical Equipment via Magnetic Nanoparticles in Pasteur Pipettes for Pathogen Detection. Anal. Biochem. 2021, 635, 114445. https://doi.org/10.1016/j.ab.2021.114445.

(44) Xie, X.; Lokugamage, K. G.; Zhang, X.; Vu, M. N.; Muruato, A. E.; Menachery, V. D.; Shi, P.-Y. Engineering SARS-CoV-2 Using a Reverse Genetic System. Nat. Protoc. 2021, 16 (3), 1761-1784. https://doi.org/10.1038/s41596-021-00491-8.

(45) Department of Health and Human Services. NIH Guidelines for Research Involving Recombinant or Synthetic Nucleic Acid Molecules. National Institutes of Health.

(46) Jang, W. S.; Lim, D. H.; Yoon, J.; Kim, A.; Lim, M.; Nam, J.; Yanagihara, R.; Ryu, S.-W.; Jung, B. K.; Ryoo, N.-H.; Lim, C. S. Development of a Multiplex Loop-Mediated Isothermal Amplification (LAMP) Assay for on-Site Diagnosis of SARS CoV-2. PloS One 2021, 16 (3), e0248042. https://doi.org/10.1371/journal.pone.0248042.

(47) Pezzi, H. M.; Niles, D. J.; Schehr, J. L.; Beebe, D. J.; Lang, J. M. Integration of Magnetic Bead-Based Cell Selection into Complex Isolations. ACS Omega 2018, 3 (4), 3908-3917. https://doi.org/10.1021/acsomega.7b01427. 
(48) Adams, N. M.; Bordelon, H.; Wang, K.-K. A.; Albert, L. E.; Wright, D. W.; Haselton, F. R. Comparison of Three Magnetic Bead Surface Functionalities for RNA Extraction and Detection. ACS Appl. Mater. Interfaces 2015, 7 (11), 6062-6069. https://doi.org/10.1021/am506374t.

(49) Schrader, C.; Schielke, A.; Ellerbroek, L.; Johne, R. PCR Inhibitors - Occurrence, Properties and Removal. J. Appl. Microbiol. 2012, 113 (5), 1014-1026. https://doi.org/10.1111/j.13652672.2012.05384.x.

(50) CDC. CDC-Preparation of Viral Transport Media.

(51) WHO Viral Transport Media (VTM).

(52) CDC Viral Transport Media.

(53) Tsui, N. B. Y.; Ng, E. K. O.; Lo, Y. M. D. Stability of Endogenous and Added RNA in Blood Specimens, Serum, and Plasma. Clin. Chem. 2002, 48 (10), 1647-1653.

(54) Martin, A.; Bouffier, L.; Grant, K. B.; Limoges, B.; Marchal, D. Real-Time Electrochemical LAMP: A Rational Comparative Study of Different DNA Intercalating and Non-Intercalating Redox Probes. The Analyst 2016, 141 (13), 4196-4203. https://doi.org/10.1039/c6an00867d.

(55) Wozniak, A.; Cerda, A.; Ibarra-Henríquez, C.; Sebastian, V.; Armijo, G.; Lamig, L.; Miranda, C.; Lagos, M.; Solari, S.; Guzmán, A. M.; Quiroga, T.; Hitschfeld, S.; Riveras, E.; Ferrés, M.; Gutiérrez, R. A.; García, P. A Simple RNA Preparation Method for SARS-CoV-2 Detection by RT-QPCR. Sci. Rep. 2020, 10 (1), 16608. https://doi.org/10.1038/s41598-020-73616-w.

(56) Haddar, C.; Verhoeven, P. O.; Bourlet, T.; Pozzetto, B.; Pillet, S. Brief Comparative Evaluation of Six Open One-Step RT-QPCR Mastermixes for the Detection of SARS-CoV-2 RNA Using a Taqman Probe. J. Clin. Virol. Off. Publ. Pan Am. Soc. Clin. Virol. 2020, 132, 104636. https://doi.org/10.1016/j.jcv.2020.104636.

(57) BGI-A Trusted Total Lab Solution for COVID-19 Pandemic https://www.bgi.com/us/bgi-covid-19total-lab-solution/ (accessed $2021-12-08$ ).

(58) GeneXpert ${ }^{\circledR}$ Prices. FIND.

(59) Lee, S.; Crulhas, B. P.; Suvakov, S.; Verkhoturov, S. V.; Verkhoturov, D. S.; Eller, M. J.; Malhi, H.; Garovic, V. D.; Schweikert, E. A.; Stybayeva, G.; Revzin, A. Nanoparticle-Enabled Multiplexed Electrochemical Immunoassay for Detection of Surface Proteins on Extracellular Vesicles. ACS Appl. Mater. Interfaces 2021, 13 (44), 52321-52332. https://doi.org/10.1021/acsami.1c14506.

(60) Hsieh, K.; Patterson, A. S.; Ferguson, B. S.; Plaxco, K. W.; Soh, H. T. Rapid, Sensitive, and Quantitative Detection of Pathogenic DNA at the Point of Care via Microfluidic Electrochemical Quantitative Loop-Mediated Isothermal Amplification (MEQ-LAMP). Angew. Chem. Int. Ed Engl. 2012, 51 (20), 4896-4900. https://doi.org/10.1002/anie.201109115.

(61) Santiago, I. Trends and Innovations in Biosensors for COVID-19 Mass Testing. ChemBioChem 2020, 21 (20), 2880-2889. https://doi.org/10.1002/cbic.202000250.

(62) Nawattanapaiboon, K.; Pasomsub, E.; Prombun, P.; Wongbunmak, A.; Jenjitwanich, A.; Mahasupachai, P.; Vetcho, P.; Chayrach, C.; Manatjaroenlap, N.; Samphaongern, C.; Watthanachockchai, T.; Leedorkmai, P.; Manopwisedjaroen, S.; Akkarawongsapat, R.; Thitithanyanont, A.; Phanchana, M.; Panbangred, W.; Chauvatcharin, S.; Srikhirin, T. Colorimetric Reverse Transcription Loop-Mediated Isothermal Amplification (RT-LAMP) as a Visual Diagnostic Platform for the Detection of the Emerging Coronavirus SARS-CoV-2. The Analyst 2021, 146 (2), 471-477. https://doi.org/10.1039/d0an01775b. 
